\title{
Mobile 2-, 3-D Radar Network Error Registration Model
}

\author{
Lei Chen ${ }^{1}$, G.H. Wang ${ }^{1}$, and Ilir F. Progri ${ }^{2}$ \\ ${ }^{1}$ Naval Aviation University, Yantai, Shandong Province, 264001, China \\ ${ }^{2}$ Giftet Inc., 5 Euclid Ave. \#3, Worcester, MA 01610, USA
}

Correspondence should be addressed to Lei Chen; chenlei_hjhy@sina.com

Received - December 19, 2015; Revised December, 20-March 5, 2016, June 10-12, 18-21 2018; Accepted June 21, 2018

Scientific Editor: Ilir F. Progri

Copyright (C) 2018 Lei Chen et al. This is an open access article distributed under the Creative Commons Attribution License, which permits unrestricted use, distribution, and reproduction in any medium, provided the original work is properly cited.

\begin{abstract}
Registration is the act or process of simultaneously entering information about radar offset biases and attitude biases of Inertial Navigation System (INS) in a common reference frame known as radar registration frame. The variables that describe the registration are defined as radar offset biases and attitude biases. The mathematical relationship that shows the formal relation among the registration variables or radar offset biases and attitude biases is defined as the registration equation(s). For mobile 2-, 3-D radar networks, in order to improve the accuracy of the registration equations, the earth-centered earth-fixed (ECEF) coordinate system is used as the intermediate frame to get to the registration frame. Since a 2-D radar model has no comprehensive 3-D information of the target when establishing the registration equations, three different methods are discussed, each method differently and uniquely pinpoints to a reference point. The latter instead of the true target coordinates is used to establish the registration equations. The errors of the reference points are unavoidable and will be embodied in the estimation errors of all the biases. The mobile 2-D radar network registration in ECEF frame can also be included in this methodology. Simulation results show that all three methods can be applied well for registration, and for a certain condition, one of them outperforms the others.
\end{abstract}

Keyword: attitude bias; offset bias; registration; 3-D radar; 2-D radar, registration equations, observability, sensitivity analysis.

\section{Introduction}

For information fusion system of radar surveillance network, registration is a prerequisite technology to be considered. Sensor registration refers to the process of estimating sensor systematic biases and compensating sensor measurements. If no registration process is made, when raw measurements of different sensors are fused directly, besides inaccurate target location estimations, data disassociation and redundant tracks will occur which are harmful to situation awareness. In order to overcome the adverse influences mentioned above, a lot of registration methods were developed [1], which can be divided into nonlinear and linearized models, respectively. All these methods select the systematic biases as state vector to establish the equations (since these equations are used for systematic bias estimation, we call them registration equations), and the major differences are the estimation algorithms. In addition, these methods are based on stationary radar networks, and the systematic biases in consideration include range, the gain of range, azimuth and elevation biases which are usually called as offset biases.

At present, the registration technologies are usually used for homogeneous sensors such as 2-, 3-D radar networks. 
However, for 2-, 3-D radar registration, few researches have been performed [2], yet it is an important complement technology in theory and practice. For 3-D radars, the 3-D reference system, especially Earth-Centered Earth-Fixed (ECEF) frame is used as the reference frame to establish the registration equations because of the high conversion accuracy and the convenient access to the real-time geographic coordinates of radars. As for 2-D radars, since the target altitude information cannot be obtained by radar network, 2-D plane frame is usually used [3], and the target altitudes are usually assumed to be zeros. This assumption omits the following presumptions: (1) the true target altitudes are not zeros; (2) the target altitudes in different radars' local coordinates are different; (3) the axes of both radars' local frames are not parallel. Hence, this assumption is harmful to the accuracy of the registration model. The same problems occur for 2-, 3-D radar network. Therefore, the selection process of the reference frame to establish the registration model with incomplete target information is crucial for 2-, 3$\mathrm{D}$ radar registration.

Another key issue for heterogeneous sensor registration is the selection of the state vector. Usually, two kinds of state vectors can be used: (1) equations which contain target states and systematic biases [2], [3]; and (2) only systematic biases are contained. However, since the existence of the dependence between the initial target states and systematic biases, the usage of the former equation set is not appropriate (high computation burden and slow convergence speed) [4]. Then the latter equations are used in the article.

Furthermore, for mobile radars, radar measurements contain attitude biases of the platform at the same time. Attitude biases occur due to the accumulated biases in the gyros in the Inertial Measurement Units (IMU) of the INS [1]. The most difficult thing for mobile radar registration is to estimate offset biases and attitude biases simultaneously since the coupling between them is inevitable. For mobile radar, Wang et al. (2013, [4]) deduced the equivalent measurement bias expressions caused by attitude biases. These expressions manifest the specific dependencies among attitude and offset biases and show that the registration equations containing both kinds of biases are unobservable. Chen et al (2014, [6]) proposed a unified registration model (URM) whose state vector only contains radar offset biases. The registration model in this situation is proved observable and the estimation results of offset biases contain the combined influence of attitude biases since each element in the state vector is in fact the composition of both kinds of biases. The equivalent measurement bias expressions are not constant functions, they vary with different target locations, and the variations are small in magnitudes compared with the invariant parts. In URM, the state equation is modeled as a constant plus a random noise. Since the dependencies among both kinds of biases are considered, URM can be applied well for mobile 3$\mathrm{D}$ radar error registration model.

The main purpose of this journal paper is to establish the registration model for mobile 2-, 3-D radar network. In order to make the registration model observable, according to URM for mobile 3-D radar registration, all the attitude biases cannot appear in the state vector which is minimized to contain only offset biases. Another key issue for registration is how to establish the registration equations. Since it is convenient to obtain mobile radars' real-time geographic coordinates through Global Position System (GPS), ECEF coordinates conversion system is selected for its higher conversion accuracy. The registration equations are established according to the principle that the true target coordinates included in both radars' raw measurements are equal when they are converted to a common reference frame, respectively. Since 2-D radar measurements do not contain the target altitudes, in order to establish the registration equations, three methods will be given in the paper, method 1 assumes that the target altitudes in 2-D radar measurement frame are zeros; Method 2 converts 3-D radar measurements to 2-D radar Cartesian coordinates to obtain the altitudes; Method 3 converts 3-D radar measurements into 2-D radar polar coordinates to establish range and azimuth registration equations, and it avoids using the elevation measurements of 2-D radar. Simulation results show that three methods can be applied for registration well; however, they all have unavoidable errors because it is the reference points instead of the true target locations that are used in the registration equations; and the errors of the reference points will be embodied in the estimations of all the biases. However, each method can be applied well in certain circumstances.

Since ECEF coordinates conversion system needs 3-D location information of the target; hence, these three models can be easily applied for 2-D radar network registration when one of the 2-D radars preset the target altitude values. 
Therefore, 2-, 3-D radar network (the target altitudes contain biases/noises) and 2-D radar network (the target altitudes are preset) registration can be included in the same category because neither situation can obtain accurate target altitude information. Our discussion includes 2-D radar network situations, where different methods for presetting target altitude values are employed. Since the target altitude has inevitable errors, the sensitivity analysis is used in the journal paper to analyze the influences of these errors on bias estimations. Simulation results show that small elevation errors have little influence on the bias estimation performance.

For length limitation, sensor location errors caused by bias errors in the navigation system and timing biases in the clocks of the sensors will be discussed specially later. It is assumed that both radars have accurate position information of themselves; they are synchronized and have the same sampling intervals. And both offset and attitude biases are assumed as constants.

This paper is organized as follows. In Sect. 2, a basic mathematical model is developed for 3-D and 2-D radar registration. Then the equivalent state equations and three different kinds of registration models according to different altitude selection methods for 2-D radar are derived in Sect.3. These methods are expanded to 2-D radar networks in Sect.4. The sensitivity analysis is performed in Sect.5 to analyze the influences of the target altitude errors on bias estimations. The test setup and simulation results of the algorithms exited by simulated track data are provided in Sect.6. Finally, in Sect.7, the conclusion of this study is given.

\section{Mathematical description}

The problem addressed in this work can be stated as follows. Consider 3-D radar A and 2-D radar B (see Fig. 1 (a)), which are installed on the $i$ th moving ship via three-axis gyrostabilized platforms, respectively, where $i=\{A, B\}$.

The geographic coordinates of the $i$ th ship are latitude $L_{s i}$, longitude $R_{s i}$, and altitude $H_{s i}$, which are known in real-time. Gyro-stabilized platform of each ship can steadily track local east-north-up (ENU) frame; however, for the bias errors in the IMU [8], the axes of the gyro-stabilized platform have a set of Eulerian angles with the corresponding axes of ENU frame. These angles are attitude biases which can be defined as yaw,
$\Delta \phi$, pitch, $\Delta \eta$, and roll bias $\Delta \psi$, respectively. For ENU frame, its origin $o$ is located at the center of the gyro-stabilized platform, where three mutually orthogonal axes $x, y$, and $z$ refer to the directions of east, north, and up, respectively. The plane xoy is horizontal. We refer to the output Cartesian coordinates of the gyro-stabilized platform as the platform frame. Radar measurements are generated in the platform frame. As shown in Fig. 1 (a), $T_{t}$ denotes the true target coordinates in space, $T_{m A}$ and $T_{m B}$ denote biased measurements of radar A and $\mathrm{B}$, respectively. Let $o_{p_{A} t}$ and $o_{p_{B} t}$ denote the projections of the true target coordinates in radar A and B platform frame, respectively. Let $o_{p_{A} T_{A}}$ and $o_{p_{B} T_{B}}$ denote the projections of radar measurements in their native platform frame.

Platform frame has the same origin with ENU; Fig. 1(b) shows the conversion from the platform frame to ENU, where $x_{p}, y_{p}$, and $z_{p}$ denote $x, y$, and $z$ axis of the platform frame, respectively, and the axes drawn in dashed lines are intermediate axes. As shown in Fig. 1(b), the transition of the target coordinates from the platform frame to ENU is accomplished by first rotating about the $y$-axis of the platform frame by the roll bias, $\Delta \psi$, then rotating about the intermediate $x$-axis by the pitch bias, $\Delta \eta$, and rotating about the final $z$-axis by the yaw bias $\Delta \phi$. Typically, the polarity definitions of $\Delta \phi$ and $\Delta \psi$ abide by the left-hand rule [17] with their respective rotation axes, and $\Delta \eta$ abides by the right-hand rule [18] (the four fingers curl in the same direction with the bias angle, then the direction of the thumb according to the rotation axis represent bias polarity. If the thumb point to the positive direction of the axis, the bias is positive, and vice versa. In this process, if the left-hand is used, it is called the left-hand rule. So does the right-hand rule).

Radar measurements are performed on the measurement frame which shares the same origin with the platform frame. However, there are azimuth and elevation biases among corresponding axes of both frames. Figure 1(c) describes 3-D radar measurements where radar locates at the origin $o$. Let $T_{m}^{\prime}$ denote the ghost target influenced by the azimuth bias only and let $T_{m}$ denote the ghost target influenced by all the biases. Let $o_{p}, o_{m}^{\prime}$, and $o_{m}$ denote the corresponding projections on the platform frame $x_{p} o y_{p}$ plane, respectively. Let $o_{e}$ denote the projection of the true target on the horizontal plane of the ENU frame. The measurements of the targets from the $i$ th radar include range, $r_{i}$, azimuth, $\theta_{i}$, (in the 
paper, true north corresponds to $\theta=0$, and the clockwise direction denotes the increment of $\theta$ ), and elevation, $\varepsilon_{i}$, for 3 $\mathrm{D}$ radar only. These measurements contain the true target position information (such as range, $r_{i t}$, azimuth, $\theta_{i t}$, and elevation, $\varepsilon_{i t}$ ); radar offset biases (such as range, $\Delta r_{i}$, the gain of range, $k_{r i}$, which arises for atmospheric refraction [9], azimuth, $\Delta \theta_{i}$, and elevation bias $\Delta \varepsilon_{i}$. The polarity definitions of $\Delta \theta_{i}$ and $\Delta \varepsilon_{i}$ are identical with $\theta$ and $\varepsilon$, respectively.); attitude biases of gyro-stabilized platform; and random measurement errors (such as range, $\delta_{r i}$, azimuth, $\delta_{\theta i}$, and elevation, $\delta_{\varepsilon i}$,errors). Random measurement errors are zeromean, Gaussian with known standard deviations.

The main objective of the paper is to establish the registration equations for mobile 2-, 3-D radar network. Registration is the act or process of simultaneously entering information about two radars' offset biases and attitude biases of INS in a common reference frame knows as the radar registration frame. The variables that describe the registration are defined as radar offset biases and attitude biases. The mathematical relationship that shows the formal relation among the registration variables or radar offset biases and attitude biases is defined as the registration equation(s). Since the attitude biases are relevant to the corresponding offset biases [4], in the article, the attitude biases are merged into the offset biases to be estimated simultaneously, that is, only offset biases will occur in the state variables. The registration equations are established according to the principle that the true target locations contained in both radars' measurements are equal when they are converted into the same frame. However, for radar network containing 2-D radar, true target altitude information cannot be obtained, in order to establish the registration equations in 3-D space, "true altitude" of the target should be obtained previously according to some principles. For this reason, it is the reference point other than the true target coordinates that is used for registration equations. The coordinates errors between the reference point and the true target location will influence both the offset and attitude biases estimation. In the paper, three methods corresponding to three different target altitude assignments are discussed. And the state vector of the registration equations only contains both radars' offset biases, as for the attitude biases, their effects are included in the corresponding offset biases. Kalman filter is used to estimate these systematic offset biases. The local ENU frame of 2-D radar is selected as the common reference frame where the registration equations are established. Also the true target coordinates included in 3-D radar measurements will be converted into this frame. The following are the main procedures:

According to [4], all the attitude biases can be equivalent to measurement errors at time instance $k$ as:

$$
\begin{aligned}
& \Delta r_{i c}(k)=0+o\left(\Delta \phi_{i}, \Delta \eta_{i}, \Delta \psi_{i}\right) \\
& \Delta \theta_{i c}(k)=\mid \begin{array}{c}
-\Delta \phi_{i}+\frac{y_{i}(k) z_{i}(k) \Delta \psi_{i}-x_{i}(k) z_{i}(k) \Delta \eta_{i}}{x_{i}^{2}(k)+y_{i}^{2}(k)} \\
+o\left(\Delta \phi_{i}, \Delta \eta_{i}, \Delta \psi_{i}\right)
\end{array} \\
& \Delta \varepsilon_{i c}(k)=\frac{-x_{i}(k) \Delta \psi_{i}-y_{i}(k) \Delta \eta_{i}}{\sqrt{x_{i}^{2}(k)+y_{i}^{2}(k)}}+o\left(\Delta \phi_{i}, \Delta \eta_{i}, \Delta \psi_{i}\right)
\end{aligned}
$$

where $\Delta r_{i c}(k), \Delta \theta_{i c}(k)$, and $\Delta \varepsilon_{i c}(k)$ denote the equivalent range, azimuth, and elevation errors of $i$ th radar converted from the attitude biases, respectively. Let $\mathbf{x}_{i}=$ $\left[x_{i}, y_{i}, z_{i}\right]^{\dagger_{i}}$ denotes the true target Cartesian coordinates in the $i$ th platform frame, since it cannot be obtained directly; it is usually approximated by the coordinates converted from the $i$ th radar raw measurements (this approximation is also used in (4a)). When (1)-(3) are used, the true target coordinates in ENU frame can be written directly without using rotation transformations which are needed for the coordinates transformation from the platform frame to ENU frame because of the attitude biases [7].

\subsection{True target coordinates in ENU frame}

The true target coordinates contained in 3-D radar measurements can be converted into 2-D radar ENU frame to establish the registration equations according to the main steps which will be discussed next.

Let the column vectors $\mathbf{x}_{i_{-} E N U}=\left[x_{i_{-} E N U}, y_{i_{-} E N U}, z_{i_{-} E N U}\right]^{\dagger}$ and $\mathbf{x}_{i_{-} E C E F}=\left[x_{i_{-} E C E F}, y_{i_{-} E C E F}, z_{i_{-} E C E F}\right]^{\dagger}$ represent the true rectangular coordinates of the target in ENU and ECEF frame, respectively, of the $i$ th radar. According to (1)-(3), we have 


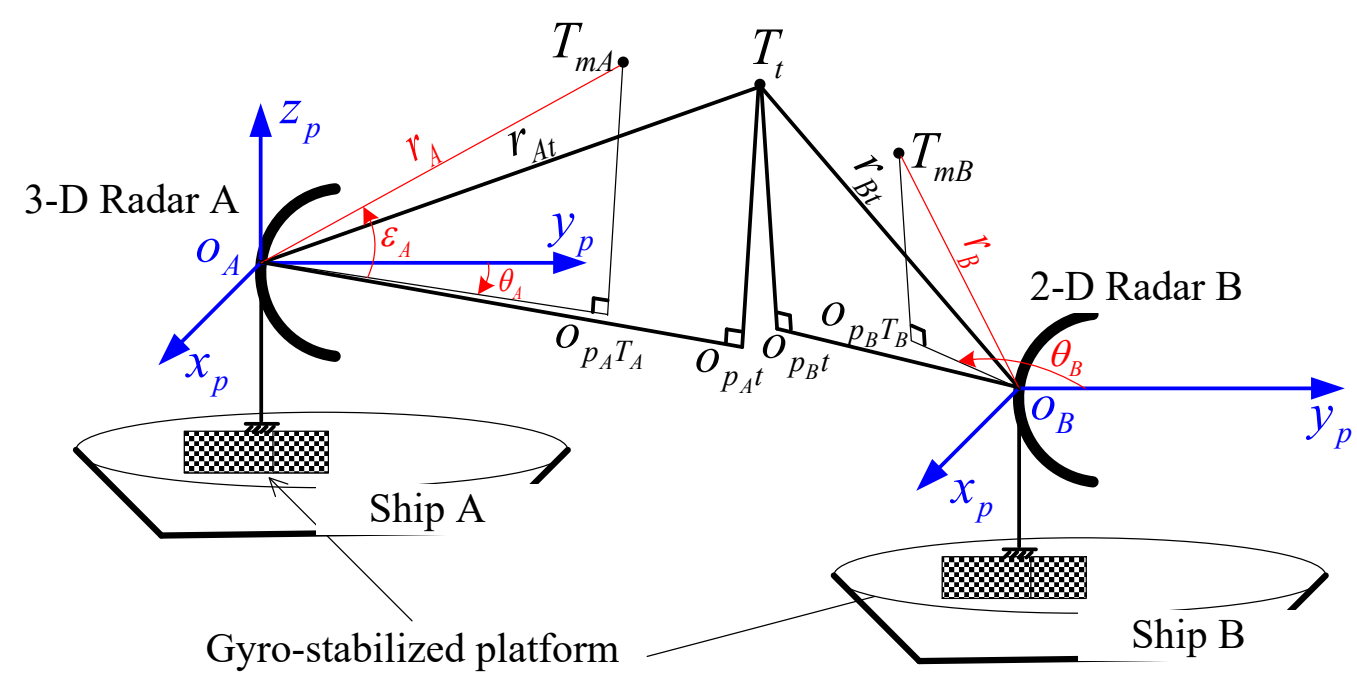

(a) The diagram of the geometry of radar and target and radar measurement.
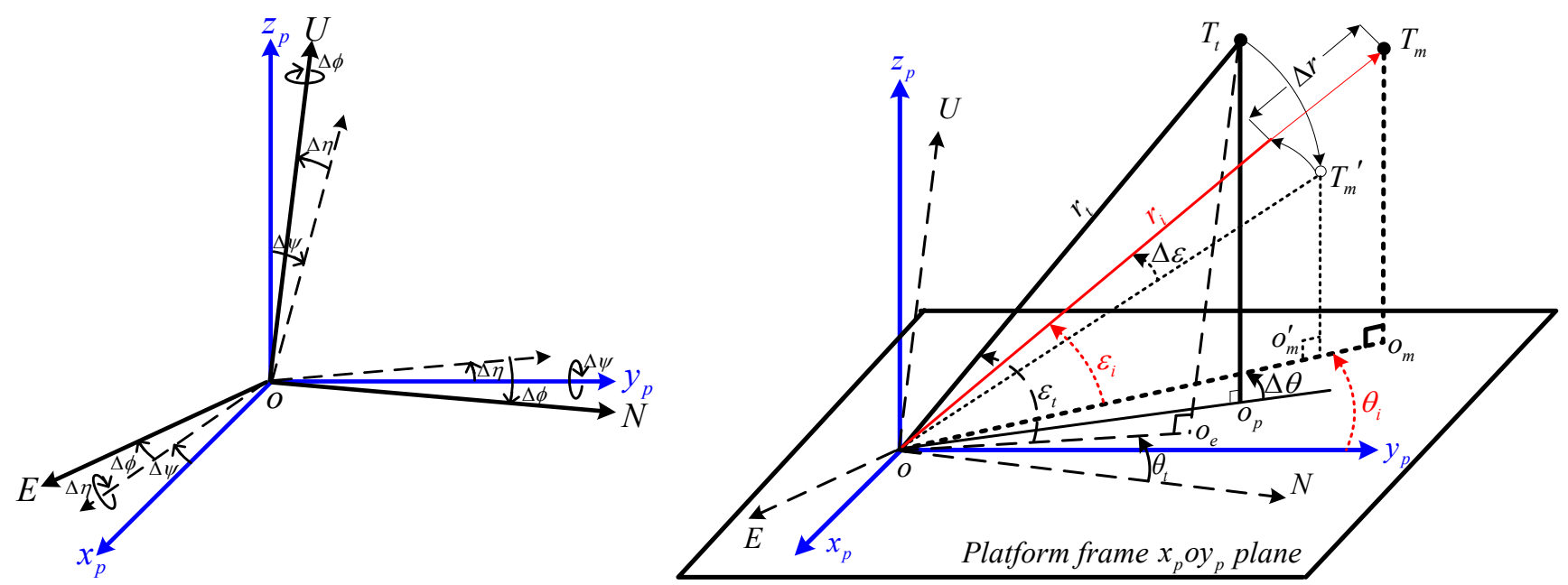

(b) Conversion from the platform frame to ENU (c) 3-D radar measurement in platform frame

FIGURE 1:3-D radar description, coordinate transformation, and measurement platform frame.

$$
\begin{gathered}
\mathbf{x}_{A_{-} E N U}=\gamma_{A}(k)\left[\mathbf{e}\left(\alpha_{A}, \beta_{A}\right)(k) \equiv\left[\begin{array}{c}
\sin \alpha_{A}(k) \cos \beta_{A}(k) \\
\cos \alpha_{A}(k) \cos \beta_{A}(k) \\
\sin \beta_{A}(k)
\end{array}\right]\right](4 \mathrm{a}) \\
\alpha_{A}(k)=\theta_{A}(k)-\Delta \theta_{A}^{\prime}(k)-\delta_{\theta A}(k) \\
\beta_{A}(k)=\varepsilon_{A}(k)-\Delta \varepsilon_{A}^{\prime}(k)-\delta_{\varepsilon A}(k) \\
\gamma_{A}(k)=r_{A}(k)-\Delta r_{A}^{\prime}-\delta_{r A}(k)
\end{gathered}
$$

where

$$
\begin{gathered}
\Delta \theta_{A}^{\prime}(k)=\Delta \theta_{A}+\Delta \theta_{A c}(k) \\
\Delta \varepsilon_{A}^{\prime}(k)=\Delta \varepsilon_{A}+\Delta \varepsilon_{A c}(k) \\
\Delta r_{A}^{\prime}=\Delta r_{A}+k_{r A}(k) r_{A t}(k)
\end{gathered}
$$

and $r_{A t}$ denotes the true target range which cannot be obtained in reality, it can be approximated by the range measurement $r_{A}$.

In [6], observability matrix $(\mathrm{OM})$ is used to prove that in the registration equation set, if the coefficients of two variables in each equation have the same proportion, and both variables always occur simultaneously in the registration equations in the form of addition, then the $\mathrm{OM}$ is singular and the system is unobservable. In [19], the concept of indistinguishability is used to testify the observability special for the stochastic system. And in appendix A, this criterion will be used to prove the same unobservable results for the conditions mentioned above. 




FIGURE 2: Algorithm flow chart for three methods.

Appendix A demonstrates that only "offset biases" should be maintained as the state variables to ensure the observability of the system, that is, the composite state vector can be written as:

$$
\boldsymbol{\beta}_{A}(k)=\left[\Delta r_{A}, k_{r A}, \Delta \theta_{A}^{\prime}(k), \Delta \varepsilon_{A}^{\prime}(k)\right]^{\dagger}
$$

where for offset biases $\Delta r_{A}, k_{r A}, \Delta \theta_{A}$, and $\Delta \varepsilon_{A}$, they are usually viewed as slowly-varying or constant models because the fast-varying parts can be classified into random measurement noises. Attitude biases of INS are also slowlyvarying variables. As for $\Delta \theta_{A}^{\prime}(k)$ and $\Delta \varepsilon_{A}^{\prime}(k)$, according to (1)-(3), when the target coordinates variations are small compared with the target coordinates in observation time interval, they can be approximately used as constants (for precise estimate, $\Delta \theta_{A}^{\prime}(k)$ and $\Delta \varepsilon_{A}^{\prime}(k)$ can be added a constant function, respectively, which use target coordinates measurements as variables to rectify the bias estimate errors). So, for simplicity, variables in (5) are all viewed as constants in the article.

Each element of $\mathbf{x}_{A_{-} E N U}$ is a nonlinear function of offset biases, attitude biases, and random measurement errors. Usually, these variables are small in magnitudes, and they can be approximated by the first-order Maclaurin series as

$$
\mathbf{x}_{A_{-} E N U}(k) \approx \mathbf{x}_{A}(k)+\mathbf{A}_{A}(k) \boldsymbol{\beta}_{A}(k)+\mathbf{C}_{A}(k) \mathbf{w}_{A}(k)
$$

where

$$
\begin{aligned}
& \mathbf{x}_{A}(k)=\left.\mathbf{x}_{A_{-} E N U}(k)\right|_{\begin{array}{l}
\boldsymbol{\beta}_{A}(k)=\mathbf{0} \\
\mathbf{w}_{A}(k)=\mathbf{0}
\end{array}} \\
& \mathbf{A}_{A}(k)=\left.\frac{\partial \mathbf{x}_{A_{E} E N U}(k)}{\partial \boldsymbol{\beta}_{A}(k)}\right|_{\begin{array}{l}
\boldsymbol{\beta}_{A}(k)=\mathbf{0} \\
\mathbf{w}_{A}(k)=\mathbf{0}
\end{array}} \\
& \mathbf{C}_{A}(k)=\left.\frac{\partial \mathbf{x}_{A_{-} E N U}(k)}{\partial \mathbf{w}_{A}(k)}\right|_{\begin{array}{l}
\boldsymbol{\beta}_{A}(k)=\mathbf{0} \\
\mathbf{w}_{A}(k)=\mathbf{0}
\end{array}}
\end{aligned}
$$

$$
\mathbf{w}_{A}(k)=\left[\delta_{r A}(k), \delta_{\theta A}(k), \delta_{\varepsilon A}(k)\right]^{\dagger}
$$

Since $\mathbf{x}_{A E N U}$ represents the true target coordinates in radar AENU frame, it should be converted into the true coordinates in the ECEF frame and ultimately in radar B ENU frame to establish the registration equations.

\subsection{True target coordinates in ECEF frame}

Given the geographic coordinates of radar $i$, the true target coordinates in ECEF can be written as [10]

$$
\mathbf{x}_{i_{-} E C E F}(k)=\mathbf{x}_{i S}(k)+\mathbf{T}_{i}(k) \mathbf{x}_{i_{-} E N U}(k)
$$

where $\mathbf{x}_{i s}$ denotes the $i$ th radar ECEF coordinates converted from its geographic coordinates, and $\mathbf{T}_{i}$ is the rotation matrix. Both variables are only correlated with the geographic coordinates of the $i$ th radar at time $k$.

\subsection{Conversion to Radar B local ENU frame}

Based on the principle that $\mathbf{x}_{A_{-} E C E F}=\mathbf{x}_{B_{-} E C E F}$, according to (7), $\mathbf{x}_{A_{-} E N U}$ can be converted to radar B local ENU frame as

$$
\mathbf{x}_{B_{-} E N U}^{A}(k)=\mathbf{T}_{B}^{-1}(k)\left[\mathbf{x}_{A_{-} E C E F}(k)-\mathbf{x}_{B S}(k)\right]
$$

where

$$
\begin{aligned}
& \mathbf{x}_{A_{-} E C E F}(k)=\mathbf{x}_{A S}(k)+\mathbf{T}_{A}(k) \tilde{\mathbf{x}}_{A}(k) \\
& \tilde{\mathbf{x}}_{A}(k)=\mathbf{x}_{A}(k)+\mathbf{A}_{A}(k) \boldsymbol{\beta}_{A}(k)+\mathbf{C}_{A}(k) \mathbf{w}_{A}(k)
\end{aligned}
$$

and the superscript $A$ in $\mathbf{x}_{B_{-} E N U}^{A}$ denotes the variable is converted from radar A measurements.

\subsection{True target coordinates expressed by radar $B$ measurements}

Similar to (4a), the target coordinates expressed by radar B measurements can be written as

$$
\begin{aligned}
& \mathbf{X}_{B_{-} E N U}(k)=\gamma_{B}(k) \mathbf{e}\left(\alpha_{B}, \varepsilon_{B}\right)(k) \\
& \alpha_{B}(k)=\theta_{B}(k)-\Delta \theta_{B}^{\prime}(k)-\delta_{\theta B}(k) \\
& \gamma_{B}(k)=r_{B}(k)-\Delta r_{B}-k_{r B} r_{B}(k)-\delta_{r B}(k) \\
& \Delta \theta_{B}^{\prime}(k)=\Delta \theta_{B}+\Delta \theta_{B c}(k)
\end{aligned}
$$

where $\Delta \theta_{B}^{\prime}(k)$ denotes the gross azimuth bias which includes the radar azimuth bias and the equivalent azimuth bias converted from attitude biases; $\varepsilon_{B}(k)$ denotes the true target gross elevation in the radar B ENU frame; $\varepsilon_{B}(k)$ excludes the influences of the attitude biases of platform B.

The registration equations can be established in rectangular 
or polar coordinates of 2-D radar B measurement frame. If the rectangular frame is used, $\varepsilon_{B}(k)$ in $(9 \mathrm{a})$ should be given in advance. Since it cannot be obtained by 2-D radar, usually three different ways can be used: (1) setting $\varepsilon_{B}(k)=0$ (corresponding to method 1); (2) converting radar A raw measurements to radar B local ENU frame to obtain the approximations (method 2); (3) presetting a value according to apriori information. If the polar frame is used (method 3 ) to establish the registration model, the true target coordinates obtained from radar A raw measurements can be converted to polar coordinates of radar B to establish the registration equations. For method 3, the elevation measurements of radar $\mathrm{B}$ need not to be preset. The algorithm flow chart for three methods is depicted in Fig. 2.

\section{Registration models}

\subsection{Equivalent measurement equations}

In fact, the only difference between method 1 and method 2 is the assignments of $\varepsilon_{B}(k)$. Method 3 is quite different because it establishes the registration equations in polar coordinates. The following are the descriptions of the three registration equations respectively.

For three methods, the state vectors and noise vectors of radar $\mathrm{B}$ are the same as

$$
\begin{aligned}
& \boldsymbol{\beta}_{B}(k)=\left[\Delta r_{B}, k_{r B}, \Delta \theta_{B}^{\prime}(k)\right]^{\dagger} \\
& \mathbf{w}_{B}(k)=\left[\delta_{r B}(k), \delta_{\theta B}(k)\right]^{\dagger}
\end{aligned}
$$

According to (10) and (11), (9a) can be approximated by the first order Maclaurin series expansion as:

$$
\mathbf{X}_{B_{-} E N U}(k) \approx \mathbf{X}_{B}(k)+\mathbf{A}_{B}(k) \boldsymbol{\beta}_{B}(k)+\mathbf{C}_{B}(k) \mathbf{w}_{B}(k)
$$

where

$$
\begin{gathered}
\mathbf{X}_{B}(k)=\left.\mathbf{X}_{B_{-} E N U}(k)\right|_{\boldsymbol{\beta}_{B}(k)=\mathbf{0}} \\
\mathbf{w}_{B}(k)=\mathbf{0}
\end{gathered}
$$

(a) Method 1 , Setting $\varepsilon_{B}(k)=0$

For method 1, substituting $\varepsilon_{B}(k)=0$ into (12), the terms of the right hand side of (12) can be written as

$$
\begin{aligned}
& \mathbf{X}_{B}(k)=\left[\begin{array}{c}
r_{B}(k)\left[\sin \theta_{B}(k) \equiv s_{B}(k)\right] \equiv a_{B}(k) \\
r_{B}(k)\left[\cos \theta_{B}(k) \equiv c_{B}(k)\right] \equiv b_{B}(k) \\
0
\end{array}\right] \\
& \mathbf{A}_{B}(k)=-\left[\begin{array}{ccc}
s_{B}(k) & a_{B}(k) & b_{B}(k) \\
c_{B}(k) & b_{B}(k) & -a_{B}(k) \\
0 & 0 & 0
\end{array}\right] \\
& \mathbf{C}_{B}(k)=-\left[\begin{array}{cc}
s_{B}(k) & b_{B}(k) \\
c_{B}(k) & -a_{B}(k) \\
0 & 0
\end{array}\right]
\end{aligned}
$$

(b) Method 2, using the converted elevation

Given the geographic coordinates of radar A and B, the raw measurements (containing errors and biases) of radar A can be converted to radar B local ENU frame which is denoted as $\mathbf{X}_{B_{-} E N U}^{A r}(k)=\left[x_{B_{-} E N U}^{A r}(k), y_{B_{-} E N U}^{A r}(k), z_{B_{-} E N U}^{A r}(k)\right]^{\dagger}$, where the superscript " $A r$ " denotes the variable is converted from radar A raw measurements. The detailed derivations of $\mathbf{X}_{B_{-} E N U}^{A r}(k)$ can be seen in Appendix B.

Since the coordinates conversion process belongs to rigid body transformation, the range of the target keeps invariant through the conversions [11]. Then, the converted elevation can be written as

$$
\varepsilon_{B}^{A r}(k)=z_{B_{-} E N U}^{A r}(k) / r_{A}(k)
$$

For method 2, substituting (16) into (9a), the first-order approximation of (9a) has the same form with (12), however these terms have different meaning, they can be written as

$$
\begin{aligned}
& \mathbf{X}_{B}(k)=r_{B}(k) \mathbf{e}\left(\theta_{B}, \varepsilon_{B}^{A r}\right)(k) \\
& \mathbf{A}_{B}(k)=\mathbf{A}_{B}(k) \cos \varepsilon_{B}^{A r}(k) \\
& \mathbf{C}_{B}(k)=\mathbf{C}_{B}(k) \cos \varepsilon_{B}^{A r}(k)
\end{aligned}
$$

The following are the same for method 1 and method 2 . According to the fact that

$$
\mathbf{X}_{B_{-} E N U}^{A}(1: 2)=\mathbf{X}_{B_{-} E N U}(1: 2)
$$

the registration equations can be established, where $\mathbf{M}(1: 2)$ denotes the first and second elements of the vector M. It should be noted that the third row equation in (20) for method 1 and 2 are deleted because for the method 1, it is obvious an inequality; and for method 2 , it is an identity since the target altitude information of radar B is obtained from radar A measurement directly.

Substituting (6a) and (14) into (20), omitting time stamp ' $k$ ' 
for brevity, the equivalent measurement equations can be written as

$$
\mathbf{T}_{B}^{-1}\left(\mathbf{X}_{A S}-\mathbf{X}_{B S}+\mathbf{T}_{A} \widetilde{\mathbf{X}}_{A}\right)=\mathbf{X}_{B}+\mathbf{A}_{B} \boldsymbol{\beta}_{B}+\mathbf{C}_{B} \mathbf{w}_{B}
$$

The system state vector can be selected as

$$
\boldsymbol{\beta}=\left[\boldsymbol{\beta}_{A}^{\dagger}, \boldsymbol{\beta}_{B}^{\dagger}\right]^{\dagger}
$$

then (21) can be rearranged as

$$
Z=\mathbf{H} \beta+\Gamma W
$$

where

$$
\begin{aligned}
& \mathbf{Z}=\mathbf{X}_{B}-\mathbf{T}_{B}^{-1}\left(\mathbf{X}_{A s}-\mathbf{X}_{B S}+\mathbf{T}_{A} \mathbf{X}_{A}\right) \\
& \mathbf{H}=\left[\mathbf{T}_{B}^{-1} \mathbf{T}_{A} \mathbf{A}_{A},-\mathbf{A}_{B}\right] \mathbf{\Gamma}=\left[\mathbf{T}_{B}^{-1} \mathbf{T}_{A} \mathbf{C}_{A},-\mathbf{C}_{B}\right] \\
& \mathbf{W}=\left[\mathbf{w}_{A}^{\dagger}, \mathbf{w}_{B}^{\dagger}\right]^{\dagger}
\end{aligned}
$$

(c) Method 3, the registration equations in the polar coordinates

This method converts the true target coordinates obtained from radar A measurements into radar B polar coordinates by using ECEF as intermediate frame, then the range and azimuth equations are established as the registration equations.

\subsubsection{The range equation}

Since the rotation matrix $\mathbf{T}_{i}$ is an orthogonal matrix, that is, $\mathbf{T}_{i}^{-1}=\mathbf{T}_{i}^{T}$, according to (8a), the true target range relative to radar B converted from radar A measurement can be written as

$$
r_{B t}^{A}(k)^{2}=x_{B_{-} E N U}^{A}{ }^{2}(k)+y_{B_{-} E N U}^{A}{ }^{2}(k)+z_{B_{-} E N U}^{A}{ }^{2}(k)
$$

Or

$$
r_{B t}^{A}(k)^{2}=\left|\mathbf{X}_{B_{-} E N U}^{A}(k)\right|^{2}=\mathbf{X}_{B_{-} E N U}^{A}(k)^{\dagger} \mathbf{X}_{B_{-} E N U}^{A}(k)
$$

Or since

$$
\mathbf{X}_{B_{-} E N U}^{A}(k)=\mathbf{T}_{B}^{-1}(k)\left[\mathbf{X}_{A_{-} E C E F}(k)-\mathbf{X}_{B S}(k)\right]
$$

and since

$$
\mathbf{X}_{A_{-} E C E F-B S}(k) \equiv \mathbf{X}_{A_{-} E C E F}(k)-\mathbf{X}_{B S}(k)
$$

or

$$
r_{B t}^{A}(k)^{2}=\mathbf{X}_{A_{-} E C E F-B S}(k)^{\dagger} \mathbf{X}_{A_{-} E C E F-B S}(k)
$$

or since

$$
\mathbf{X}_{A_{-} E C E F}(k)=\mathbf{X}_{A S}(k)+\mathbf{T}_{A}(k) \mathbf{X}_{A_{-} E N U}(k)
$$

$$
\mathbf{X}_{A s-B S}(k)=\mathbf{X}_{A S}(k)-\mathbf{X}_{B S}(k)
$$

then

$$
r_{B t}^{A}(k)^{2}=r_{A t}(k)^{2}+\mid \begin{gathered}
\left|\mathbf{X}_{A s-B s}(k)\right|^{2}+ \\
2 \mathbf{X}_{A s-B S}(k)^{\dagger} \mathbf{T}_{A}(k) \mathbf{X}_{A_{-} E N U}(k)
\end{gathered}
$$

where

$$
r_{i t}(k)^{2}=x_{i_{-} E N U}{ }^{2}(k)+y_{i_{-} E N U}{ }^{2}(k)+z_{i_{-} E N U}{ }^{2}(k)
$$

or

$$
r_{i t}(k)^{2}=\left[r_{i}(k)-\Delta r_{i}-k_{r i} r_{i t}(k)-\delta_{r i}(k)\right]^{2}
$$

The right hand side of (25b) can be approximated by the first-order Maclaurin series expansion as

$$
r_{i t}(k)^{2} \approx r_{i}(k)^{2}-2 r_{i}(k)\left[\Delta r_{i}+k_{r i} r_{i t}(k)+\delta_{r i}(k)\right]
$$

The second term of the right hand side of (24h) can be approximated by the first-order Maclaurin series expansion as

$$
\mathbf{X}_{A_{-} E N U}(k)=\widetilde{\mathbf{X}}_{A}(k)
$$

According to the fact that

$$
r_{B t}^{A}(k)^{2}=r_{B t}(k)^{2}
$$

substituting (24h), (26), and (27) into (28), the range equation can be written as

$$
\begin{aligned}
r_{B t}^{A}(k)^{2} & =\mathbf{y}(k) \boldsymbol{\beta}(k)+\mathbf{z}(k) \mathbf{W}(k) \\
r_{B t}^{A}(k)^{2} & =\mid \begin{array}{c}
0.5\left[\left|\mathbf{X}_{A s-B S}(k)\right|^{2}+r_{A}(k)^{2}-r_{B}(k)^{2}\right] \\
+\mathbf{X}_{A S-B S}(k)^{\dagger} \mathbf{T}_{A}(k) \mathbf{X}_{A}(k)
\end{array}
\end{aligned}
$$

$\mathbf{x}_{A} \equiv\left[\begin{array}{c}r_{A}(k) \\ r_{A}(k)^{2} \\ 0 \\ 0\end{array}\right]^{\dagger} ; \mathbf{x}_{B} \equiv-\left[\begin{array}{c}r_{B}(k) \\ r_{B}(k)^{2} \\ 0\end{array}\right]^{\dagger}$

$\mathbf{y}(k)=\left[\boldsymbol{\Sigma}+\mathbf{x}_{\boldsymbol{A}}, \mathbf{x}_{B}\right]$

$$
\mathbf{z}(k)=\left[\begin{array}{c}
\boldsymbol{\Sigma} \\
-r_{B}(k) \\
0
\end{array}\right]^{\dagger}
$$

$$
\boldsymbol{\Sigma}=\left[\mathbf{X}_{B S}(k)-\mathbf{X}_{A S}(k)\right]^{\dagger} \mathbf{T}_{A}(k) \mathbf{C}_{A}(k)
$$

\subsubsection{The azimuth equation}

Similar to (28), the azimuth equation can be established as

$$
\sin \left[\theta_{B t}(k)\right]=\frac{x_{B_{-} E N U}^{A}(k)}{\sqrt{x_{B_{-} E N U}^{A}{ }^{2}(k)+y_{B_{-} E N U}^{A}{ }^{2}(k)}}
$$

where 


$$
\theta_{B t}(k)=\theta_{B}(k)-\Delta \theta_{B}^{\prime}(k)-\delta_{\theta B}(k)
$$

Substituting (6a) into (30), using the first-order Maclaurin series expansion for both sides of (30), we have

$$
\begin{aligned}
& \sin \left[\theta_{B}(k)\right]-\frac{x}{\sqrt{x^{2}+y^{2}}}=\boldsymbol{a}_{\boldsymbol{A}}(k)+\boldsymbol{a}_{\boldsymbol{B}}(k)+\boldsymbol{b}_{\boldsymbol{A}}(k)+\boldsymbol{b}_{\boldsymbol{B}}(k) \\
& \boldsymbol{a}_{\boldsymbol{A}}(k)=\left[\frac{\dot{x}_{\boldsymbol{\beta}}}{\sqrt{x^{2}+y^{2}}}-\frac{x^{2} \dot{x}_{\boldsymbol{\beta}}+x y \dot{y}_{\boldsymbol{\beta}}}{\left[x^{2}+y^{2}\right]^{3 / 2}}\right] \boldsymbol{\beta}_{A}(k) \\
& \boldsymbol{a}_{\boldsymbol{B}}(k)=\left\{0,0, \cos \left[\theta_{B}(k)\right]\right\} \boldsymbol{\beta}_{B}(k) \\
& \boldsymbol{b}_{\boldsymbol{A}}(k)=\left[\frac{\dot{x}_{\mathbf{w}}}{\sqrt{x^{2}+y^{2}}}-\frac{x^{2} \dot{x}_{\mathbf{w}}+x y \dot{y}_{\mathbf{w}}}{\left[x^{2}+y^{2}\right]^{3 / 2}}\right] \mathbf{w}_{A}(k) \\
& \boldsymbol{b}_{\boldsymbol{B}}(k)=\left\{0, \cos \left[\theta_{B}(k)\right]\right\} \mathbf{w}_{B}(k)
\end{aligned}
$$

and

$$
\begin{aligned}
& g=\left.g_{B_{-} E N U}^{A}(k)\right|_{\substack{\boldsymbol{\beta}_{A}(k)=\mathbf{0}, \mathbf{w}_{A}(k)=\mathbf{0}}}, \dot{g}_{\boldsymbol{\beta}}=\left.\frac{\partial g_{B_{-} E N U}^{A}(k)}{\partial \boldsymbol{\beta}_{A}}\right|_{\begin{array}{l}
\boldsymbol{\beta}_{A}(k)=\mathbf{0} \\
\mathbf{w}_{A}(k)=\mathbf{0}
\end{array}} \\
& \dot{g}_{\mathbf{w}}=\left.\frac{\partial g_{B_{B} E N U}^{A}(k)}{\partial \mathbf{w}_{A}}\right|_{\begin{array}{l}
\boldsymbol{\beta}_{A}(k)=\mathbf{0} \\
\mathbf{w}_{A}(k)=\mathbf{0}
\end{array}},(g=x, y, z)
\end{aligned}
$$

Then (29) and (31) can be combined and rewritten as the equivalent measurement equations.

\subsection{The state equations}

In order to make the registration equations observable, the equivalent azimuth and elevation biases are modeled as a constant plus a noise, respectively [6], where the noise arises from the variations of the target locations. Assuming that the target velocities in $x, y$, and $z$ directions of the $i$ th radar ENU frame have zero mean Gaussian distributions, with the standard deviations are $\sigma_{i v_{x}}, \sigma_{i v_{y}}$, and $\sigma_{i v_{z}}$, respectively. Also, the target velocities in different radar ENU frames are assumed independent. According to (1)-(3), (6), (7), and (9c), omitting the subscript $i$ and time stamp $k$ for brevity, the variances of the equivalent biases can be written as

$$
\sigma_{\Delta \theta^{\prime}}^{2}=\operatorname{Var}\left\{\Delta \theta^{\prime}\right\}
$$

or since

$$
\Delta \theta^{\prime}=a_{x} v_{x}+a_{y} v_{y}+a_{z} v_{z}
$$

then

$$
\sigma_{\Delta \theta^{\prime}}^{2}=a_{x}^{2} \operatorname{Var}\left\{v_{x}\right\}+a_{y}^{2} \operatorname{Var}\left\{v_{y}\right\}+a_{z}^{2} \operatorname{Var}\left\{v_{z}\right\}
$$

Or since

$$
\operatorname{Var}\left\{v_{i}\right\}=\sigma_{i}^{2} ; \quad i=\left\{v_{x}, v_{y}, v_{z}\right\}
$$

then

$$
\sigma_{\Delta \theta^{\prime}}^{2}=a_{x}^{2} \sigma_{v_{x}}^{2}+a_{y}^{2} \sigma_{v_{y}}^{2}+a_{z}^{2} \sigma_{v_{z}}^{2}
$$

where

$$
\begin{aligned}
& a_{x}=\frac{\left[-\left(x^{2}+y^{2}\right) z \Delta \eta-2 x(y z \Delta \psi-x z \Delta \eta)\right] T R_{s p}}{\left(x^{2}+y^{2}\right)^{2}} \\
& a_{y}=\frac{\left[\left(x^{2}+y^{2}\right) z \Delta \psi-2 y(y z \Delta \psi-x z \Delta \eta)\right] T R_{s p}}{\left(x^{2}+y^{2}\right)^{2}} \\
& a_{z}=\frac{\left[\left(x^{2}+y^{2}\right) y \Delta \psi-\left(x^{2}+y^{2}\right) x \Delta \eta\right] T R_{s p}}{\left(x^{2}+y^{2}\right)^{2}}
\end{aligned}
$$

Similarly,

$$
\begin{aligned}
& \sigma_{\Delta \varepsilon^{\prime}}^{2}=\underbrace{\operatorname{Var}\left\{a_{x} v_{x}+a_{y} v_{y}\right\}}_{a_{x}^{2} \operatorname{Var}\left\{v_{x}\right\}+a_{y}^{2} \operatorname{Var}\left\{v_{y}\right\}}=\underbrace{a_{x}^{2} \sigma_{v_{x}}^{2}+a_{y}^{2} \sigma_{v_{y}}^{2}}_{\operatorname{Var}\left\{\Delta \varepsilon^{\prime}\right\}} \\
& a_{x}=\frac{\left(-y^{2} \Delta \psi+x y \Delta \eta\right) T R_{s p}}{\left(x^{2}+y^{2}\right)^{3 / 2}}
\end{aligned}
$$

Then (29) and (31) can be combined and rewritten as the equivalent measurement equations.

\subsection{The state equations}

In order to make the registration equations observable, the equivalent azimuth and elevation biases are modeled as a constant plus a noise, respectively [6], where the noise arises from the variations of the target locations. Assuming that the target velocities in $x, y$, and $z$ directions of the $i$ th radar ENU frame have zero mean Gaussian distributions, with the standard deviations are $\sigma_{i v_{x}}, \sigma_{i v_{y}}$, and $\sigma_{i v_{z}}$, respectively. Also, the target velocities in different radar ENU frames are assumed independent. According to (1)-(3), (6), (7), and (9c), omitting the subscript $i$ and time stamp $k$ for brevity, the variances of the equivalent biases can be written as

$$
\sigma_{\Delta \theta^{\prime}}^{2}=\operatorname{Var}\left\{\Delta \theta^{\prime}\right\}
$$

or since

$$
\Delta \theta^{\prime}=a_{x} v_{x}+a_{y} v_{y}+a_{z} v_{z}
$$

then

$$
\sigma_{\Delta \theta^{\prime}}^{2}=a_{x}^{2} \operatorname{Var}\left\{v_{x}\right\}+a_{y}^{2} \operatorname{Var}\left\{v_{y}\right\}+a_{z}^{2} \operatorname{Var}\left\{v_{z}\right\}
$$

Or since

$$
\operatorname{Var}\left\{v_{i}\right\}=\sigma_{i}^{2} ; \quad i=\left\{v_{x}, v_{y}, v_{z}\right\}
$$

then 


$$
\sigma_{\Delta \theta^{\prime}}^{2}=a_{x}^{2} \sigma_{v_{x}}^{2}+a_{y}^{2} \sigma_{v_{y}}^{2}+a_{z}^{2} \sigma_{v_{z}}^{2}
$$

where

$$
\begin{aligned}
& a_{x}=\frac{\left[-\left(x^{2}+y^{2}\right) z \Delta \eta-2 x(y z \Delta \psi-x z \Delta \eta)\right] T R_{s p}}{\left(x^{2}+y^{2}\right)^{2}} \\
& a_{y}=\frac{\left[\left(x^{2}+y^{2}\right) z \Delta \psi-2 y(y z \Delta \psi-x z \Delta \eta)\right] T R_{s p}}{\left(x^{2}+y^{2}\right)^{2}} \\
& a_{z}=\frac{\left[\left(x^{2}+y^{2}\right) y \Delta \psi-\left(x^{2}+y^{2}\right) x \Delta \eta\right] T R_{S p}}{\left(x^{2}+y^{2}\right)^{2}}
\end{aligned}
$$

Similarly,

$$
\begin{aligned}
& \sigma_{\Delta \varepsilon^{\prime}}^{2}=\underbrace{\operatorname{Var}\left\{a_{x} v_{x}+a_{y} v_{y}\right\}}_{a_{x}^{2} \operatorname{Var}\left\{v_{x}\right\}+a_{y}^{2} \operatorname{Var}\left\{v_{y}\right\}}=\underbrace{a_{x}^{2} \sigma_{v_{x}}^{2}+a_{y}^{2} \sigma_{v_{y}}^{2}}_{\operatorname{Var}\left\{\Delta \varepsilon^{\prime}\right\}} \\
& a_{x}=\frac{\left(-y^{2} \Delta \psi+x y \Delta \eta\right) T R_{s p}}{\left(x^{2}+y^{2}\right)^{3 / 2}} \\
& a_{y}=\frac{\left(-x^{2} \Delta \eta+x y \Delta \psi\right) T R_{s p}}{\left(x^{2}+y^{2}\right)^{3 / 2}}
\end{aligned}
$$

Similarly,

$$
\begin{aligned}
& \sigma_{\Delta \theta^{\prime} \Delta \varepsilon^{\prime}}^{2}=\sigma_{\Delta \varepsilon^{\prime} \Delta \theta^{\prime}}^{2}=\underbrace{\mathrm{E}\left\{\Delta \theta^{\prime} \Delta \varepsilon^{\prime}\right\}=\mathrm{E}\left\{a_{x}^{2} v_{x}^{2}+a_{y}^{2} v_{y}^{2}\right\}}_{a_{x}^{2} \sigma_{v_{x}}^{2}+a_{y}^{2} \sigma_{v y}^{2}} \\
& a_{x}^{2}=\frac{\left[\left(x^{2}-y^{2}\right) z \Delta \eta-2 x y z \Delta \psi\right]\left(-y^{2} \Delta \psi+x y \Delta \eta\right) T R_{s p}{ }^{2}}{\left(x^{2}+y^{2}\right)^{7 / 2}} \\
& a_{y}^{2}=\frac{\left[\left(x^{2}-y^{2}\right) z \Delta \psi+2 x y z \Delta \eta\right]\left(-x^{2} \Delta \eta+x y \Delta \psi\right) T R_{s p} p^{2}}{\left(x^{2}+y^{2}\right)^{7 / 2}}
\end{aligned}
$$

where $T R_{s p}$ denotes radar scanning period [12]; $\operatorname{Var}[X]$ and $\mathrm{E}[X]$ denote the variance and expectation of $X$; and $\{x, y, z\}$ in (32)-(34) denote the true target $\{x, y, z\}-$ coordinates, respectively, in $i$ th radar ENU frame, which can be approximated by using radar measurements. In the formulas $\Delta \eta$ and $\Delta \psi$ can also be approximated by their possible maximum values $\Delta \eta_{\max }$ and $\Delta \psi_{\max }$, respectively.

Then, similar to URM for 3-D radar registration [6], the state equations can be written as

$$
\boldsymbol{\beta}(k+1)=\boldsymbol{\beta}(k)+\mathbf{w}_{\boldsymbol{\beta}}(k)
$$

where $\mathbf{w}_{\boldsymbol{\beta}}(k)$ represents the equivalent noise vector, and

$$
\mathbf{w}_{\boldsymbol{\beta}}(k)=\left[0,0, w_{\Delta \theta_{A}^{\prime}}, w_{\Delta \varepsilon_{A}^{\prime}}, 0,0, w_{\Delta \theta_{B}^{\prime}}\right]^{\dagger}
$$

It should be noted that since the effects of the attitude biases of the platform are only to make the measurements rotate, but the range between radar and the target will not be affected, then the range and gain of the range biases remain constants, so, zero entries appear in (36). The covariance matrix of $\mathbf{w}_{\boldsymbol{\beta}}(k)$ can be written as

$$
\mathbf{Q}=\operatorname{diag}\left(\left[\mathbf{Q}_{\boldsymbol{\beta}_{A}}, \mathbf{Q}_{\boldsymbol{\beta}_{B}}\right]\right)
$$

where $\operatorname{diag}(\boldsymbol{x})$ denotes that the elements of vector $\boldsymbol{x}$ are on the diagonal of the matrix $\mathbf{Q}$, and the other elements of $\mathbf{Q}$ are zeros as

$$
\begin{aligned}
\mathbf{Q}_{\boldsymbol{\beta}_{A}} & =\left[\begin{array}{cccc}
0 & 0 & 0 & 0 \\
0 & 0 & 0 & 0 \\
0 & 0 & \sigma_{\Delta \theta_{A}^{\prime}}^{2} & \sigma_{\Delta \theta_{A}^{\prime} \Delta \varepsilon_{A}^{\prime}}^{2} \\
0 & 0 & \sigma_{\Delta \theta_{A}^{\prime} \Delta \varepsilon_{A}^{\prime}}^{2} & \sigma_{\Delta \varepsilon_{A}^{\prime}}^{2}
\end{array}\right] \\
\mathbf{Q}_{\boldsymbol{\beta}_{B}} & =\left[\begin{array}{ccc}
0 & 0 & 0 \\
0 & 0 & 0 \\
0 & 0 & \sigma_{\Delta \theta_{B}^{\prime}}^{2}
\end{array}\right]
\end{aligned}
$$

Then the first-order approximation of the equivalent measurement equations (registration equations) and the state equations are established, and Kalman filter can be used to obtain the equivalent bias estimations.

\section{Expansion to 2-D radar network registration}

Usually the registration equations for 2-D radar network are established in 2-D plane. In this situation, local coordinates of different radars are assumed parallel and the target altitudes are assumed to be zeros. However, both assumptions are inaccurate. In fact, 3-D coordinate frame using ECEF coordinates conversion is more preferable when knowing the target altitude in one of the 2-D radars' local frame. In this sense, 2-D radar network registration is equivalent to 3-D and 2-D radar situation in essence since both situations cannot obtain accurate target altitude information.

Herein, method 3 can be used for 2-D radar network registration because the target altitude is not needed for the second 2-D radar. The registration equations for both situations are totally in the same form (see (29), (31), and (35)). The only difference is that the state vector and noise vector of radar A do not contain elevation terms and the corresponding column vectors in the coefficient matrix of the registration equations are deleted.

Since the target altitude cannot be obtained accurately, however, it is critical for the bias estimation, the calculation 
method and the influence of its error will be discussed next.

\section{Performance analysis}

Since the registration method above for 2-D and 3-D radar network is the same with 2-D radar network in essence, the following analyses are based on the 2-D radar network for its tougher conditions.

\subsection{Computation of target elevation}

\subsubsection{Modeling}

In the equivalent measurement equations (23) and (25b), the true target elevation in radar A ENU frame is needed, which cannot be obtained by single 2-D radar. However, it can be obtained geometrically by using radar A range and azimuth measurements and radar $\mathrm{B}$ range measurement. The main procedures are as follows.

Since the accurate radar geographic coordinates can be obtained in real-time, the coordinates of radar B in radar A local ENU frame can be obtained by using (8a). The conversions from ENU to ECEF frame are rotation and translation, they belong to rigid motion, so, when radar B and the target coordinates are converted to radar A ENU frame, the range between them keeps invariable. Their geometric relations are depicted in Fig.3.

Figure 3(a) denotes the local ENU frame of radar A, and $A$, $B$ denote radar $\mathrm{A}$ and radar $\mathrm{B}$, respectively. Radar A locates at the origin. $T$ denotes the target. $O_{1}$ and $O_{B}$ denote the projection of the target and radar $\mathrm{B}$ in the horizontal plane $N_{A} A E_{A}$, respectively. It is assumed that in case $1, O_{1}$ doesnot fall into the line $A O_{B}$, otherwise, it will be described in Fig. 3 (b) and discussed in case 2. The line $\mathrm{BO}_{2}$ is parallel with the horizontal plane and intersects the line $O_{1} T$ at $O_{2} . \theta_{A t}$ denotes the true target azimuth in radar A ENU frame.

Case 1: $O_{1}$ does not fall into the line $A O_{B}$

As shown in Fig. 3(a), in the horizontal plane $N_{A} A E_{A}$, we define

$$
\angle O_{1} A O_{B} \equiv \alpha=\theta_{A}^{B s}-\theta_{A t} \in[0,2 \pi)
$$

where, $\theta_{A}^{B s}$ denotes the true azimuth of radar B in the local ENU frame of radar A, that is, $\angle N_{A} A O_{B}$.

According to the cosine theorem, in $\triangle O_{1} A O_{B}$,

$$
\cos \alpha=\frac{\left|A O_{1}\right|^{2}+\left|A O_{B}\right|^{2}-\left|O_{1} O_{B}\right|^{2}}{2\left|A O_{1}\right|\left|A O_{B}\right|}=c_{\alpha}
$$

where

$$
\begin{aligned}
& \left|A O_{1}\right|=\sqrt{r_{A t}^{2}-\left|O_{1} T\right|^{2}}=x \\
& \left|A O_{B}\right|=\sqrt{\left(x_{A}^{B S}\right)^{2}+\left(y_{A}^{B S}\right)^{2}}=y \\
& \left|O_{1} O_{B}\right|=\sqrt{r_{B t}^{2}-\left(\left[\left|O_{1} T\right| \equiv u\right]-z_{A}^{B S}\right)^{2}}=z \\
& |A B|=\sqrt{\left(x_{A}^{B S}\right)^{2}+\left(y_{A}^{B S}\right)^{2}+\left(z_{A}^{B S} \equiv t\right)^{2}}=w \\
& \left(x_{A}^{B S}, y_{A}^{B S}, z_{A}^{B S}\right)^{T}=\mathbf{X}_{A}^{B S}=\mathbf{T}_{A}^{-1}\left(\mathbf{X}_{B S}-\mathbf{X}_{A s}\right)
\end{aligned}
$$

Substituting (40)-(43) into (39), (39) can be rearranged as

$$
\mid \begin{gathered}
4\left(t^{2}+y^{2} c_{\alpha}^{2}\right) u^{2}-4 t\left(w^{2}+r_{A t}^{2}-r_{B t}^{2}\right) u \\
-4 y^{2} r_{A t}^{2} c_{\alpha}^{2}+\left(w^{2}+r_{A t}^{2}-r_{B t}^{2}\right)
\end{gathered}=0
$$

According to Vieta's formulas, the solutions of (45) have the form of

$$
u \equiv\left|O_{1} T\right|=\frac{-b}{2 a} \pm \frac{\sqrt{b^{2}-4 a c}}{2 a}
$$

where

$$
\begin{aligned}
& \frac{-b}{2 a}=\frac{z_{A}^{B S}\left(|A B|^{2}+r_{A t}^{2}-r_{B t}^{2}\right)}{2\left[\left(z_{A}^{B S}\right)^{2}+\left|A O_{B}\right|^{2} \cos ^{2} \alpha\right]} \\
& \frac{\sqrt{b^{2}-4 a c}}{2 a}=\frac{\sqrt{C}}{2\left[\left(z_{A}^{B S}\right)^{2}+\left|A O_{B}\right|^{2} \cos ^{2} \alpha\right]} \\
& C=r_{e q}\left|A O_{B}\right|^{2} \cos ^{2} \alpha \\
& r_{e q}=4 r_{A t}^{2}\left[\left(z_{A}^{B S}\right)^{2}+\left|A O_{B}\right|^{2} \cos ^{2} \alpha\right]-\left(|A B|^{2}+r_{A t}^{2}-r_{B t}^{2}\right)
\end{aligned}
$$

According to (46), two different solutions for (45) will be generated simultaneously. Actually, only one solution is the true target altitude, and the other one is the mirror point. Which one should be maintained is determined by the actual conditions.

Then, the true elevation values can be obtained by

$$
\varepsilon_{A t}=\sin ^{-1}\left(\left|O_{1} T\right| / r_{A t}\right)
$$






(a) Case 1

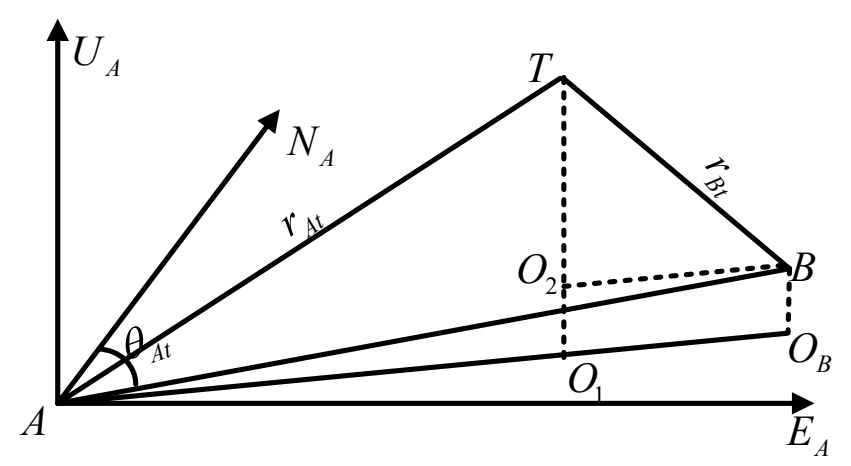

(b) Case 2

FIGURE 3: The geometry of radar and target in radar A local ENU frame.

Case 2: $O_{1}$ falls into the line $A O_{B}$, that is, $\angle O_{1} A O_{B}=$ 0 or $\pi$

As shown in Fig. 3(b), in this situation, $\operatorname{radar} \mathrm{A}, \mathrm{B}$, target $T$, and the projection $O_{1}$ and $O_{B}$ are coplanar.

In this case, according to the span of $\angle T A B+\angle B A O_{B}$, another two different situations should be considered.

1) $\angle T A B+\angle B A O_{B}<\pi / 2$

Then

$$
\varepsilon_{A t}(k)=\angle T A B+\angle B A O_{B}
$$

where $\angle T A B$ can be obtained by using cosine theorem in $\triangle T A B$ as

$$
\angle T A B=\cos ^{-1}\left(\frac{|A B|^{2}+r_{A t}^{2}-r_{B t}^{2}}{2 r_{A t}|A B|}\right)
$$

and

$$
\angle B A O_{B}=\tan ^{-1}\left[z_{A}^{B S} / \sqrt{\left(x_{A}^{B S}\right)^{2}+\left(y_{A}^{B S}\right)^{2}}\right]
$$

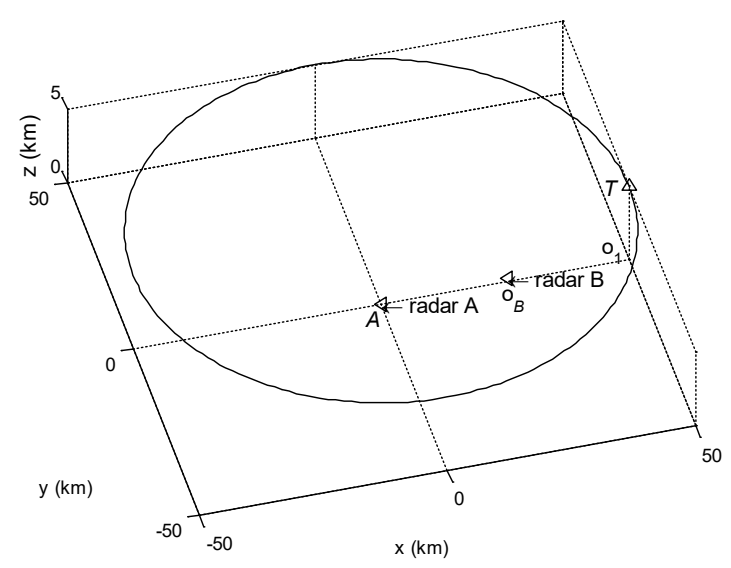

(a) The geometry of radar and target track

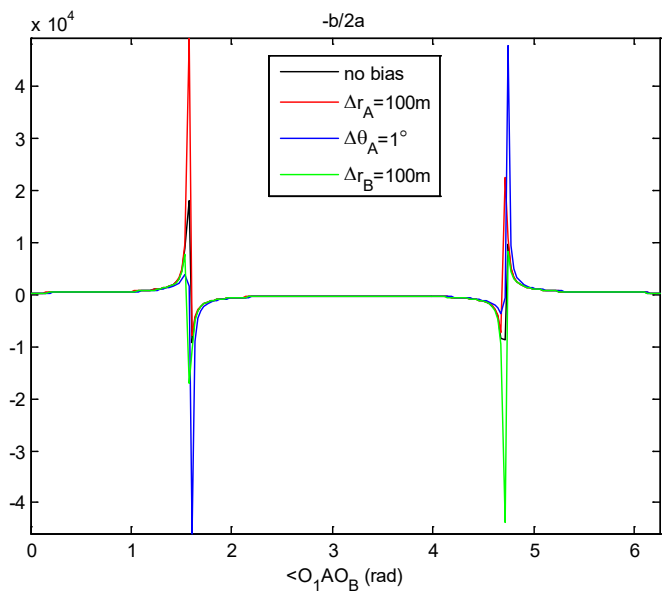

(b) Variation of the first term in the target altitude solution caused by different target location (i.e. results of (47))

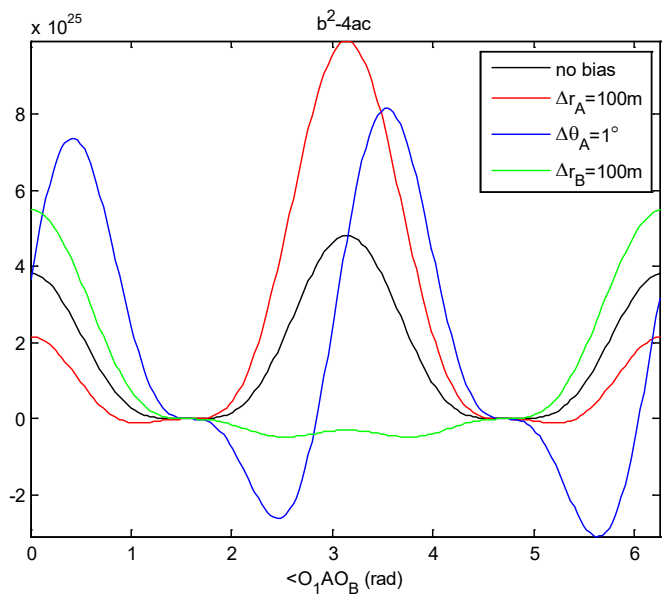

(c) Variation of the term which has the greatest impact on target altitude calculation results caused by different target location (i.e. results of (49)) 




(d) Variation of the second term in the target altitude solution expression caused by different target location (i.e. the square of the results of (48))

FIGURE 4: The calculation results analysis of the target elevation.

2) $\angle T A B+\angle B A O_{B}>\pi / 2$

$$
\varepsilon_{A t}(k) \approx \pi-\left(\angle T A B+\angle B A O_{B}\right)
$$

Usually, the overhead targets cannot be seen by radars, so, the situation when $\angle T A B+\angle B A O_{B}=\pi / 2$ will not be considered.

\subsubsection{Calculation results analysis}

In order to analyze the computational method above, a set of comparative tests are simulated in a common scenario. Assuming that the geographic coordinates of radar A and $\mathrm{B}$ are $\left[40^{\circ}, 113^{\circ}, 100 \mathrm{~m}\right]$ and $\left[40^{\circ}, 113.3^{\circ}, 300 \mathrm{~m}\right]$, respectively. As shown in Fig. 4(a), radar A locates at fusion center.

The projections of radar $\mathrm{B}$ and the target on the horizontal plane are denoted by $O_{B}$ and $O_{1}$, respectively. The line $A O_{B}$ is defined as the positive direction of $x$-axis. The target moves with constant speed around radar A, and the distances between them keep invariant as $50 \mathrm{~km}$. The altitudes of the target keep invariant as $5 \mathrm{~km}$.

The initial location of the target satisfies $\angle O_{1} A O_{B}=0$. 200 observation points are selected homogenously on the target track. Selecting $\angle O_{1} A O_{B}$ as variable, four cases are compared:(1) neither systematic biases nor random noises are included in measurements; (2) only $100 \mathrm{~m}$ range bias of radar A is contained; (3) only $1^{\circ}$ azimuth bias of radar $\mathrm{A}$ is contained; (4) only $100 \mathrm{~m}$ range bias of radar B is contained. Calculation results are shown in Fig.4.

In Fig. 4, the black lines represent case (1); the red lines represent case (2); the blue lines represent case (3); and the green lines represent case (4). Case (a) represents the geometry of radar and the target track; case (b) represents the calculation results of (47) at different target location; case (c) represents the results of (49); and case (d) represents the square of the result of (48). The horizontal axes of (b)-(d) represent $\angle O_{1} A O_{B}$ in radian.

Figure 4 shows that in most situations, the measurement errors has little effects on the solutions of (47), however, the effects on (48) are big, even very small measurement errors, especially for azimuth errors, which are the same as random measurement noises in magnitudes can affect the solution of $C$ in (48) greatly, even change its polarity. This will influence the altitude results greatly because $C$ is the critical factor for computation. It is also shown that when the target near $y$-axis, the sign of the second term of (46) will change suddenly, which always leads to miscalculations.

According to the analyses above, the computational method for solving the target altitude has the following features: (1) when measurements have no errors (biases and noises), the solutions are accurate; (2) when the target near $y$-axis, the sign of the second term of (46) will change suddenly, and the wrong solution is inclined to be selected; (3) noises or biases, especially for azimuth measurements, even if they have the same magnitudes with random measurement noises can lead to unbelievable results. Since the measurement errors are unavoidable, the computational method is unacceptable.

It would be better to preset the target altitude according to the target attributes or the information provided by other sensors. If no such information can be used, an empirical formula is usually adopted which considers the earth curvature as [15]

$$
h=\left\{\begin{array}{lr}
6000, & r^{\mathrm{ii}}<4.12 \sqrt{h_{s}{ }^{\mathrm{iii}}} \\
6000-0.5\left(\frac{r}{4.12}-\sqrt{h_{s}}\right)^{2}, & r \geq 4.12 \sqrt{h_{s}}
\end{array}\right.
$$

where $h$ denotes the target altitude in radar local ENU frame with meter as its unit, $h_{s}$ denotes the absolute altitude of radar with meter as its unit; and $r$ denotes the target range with kilometer as its unit. The principles of (55) are as follows: (1) when the target locates within the visibility range of radar, 
the altitude of the target in radar ENU frame is assumed as 6; (2) otherwise, the mean value of the lowest absolute altitude visible for radar which related with the target range and 12 is selected as the target absolute altitude.

Since the errors of the preset target altitude values are unavoidable, their influences on radar systematic bias estimations will be analyzed next.

\subsection{Sensitivity analysis for bias estimations}

Sensitivity analysis method [13], [14] is used here to analyze the influences of the elevation errors on the systematic bias estimations. Since the sensitivity analysis is derived from the linear system, and the registration model used in the paper is first-order linearized model, when the preset elevation values deviate from the true values slightly, the method is applicable. It is implemented by computing the derivative of the covariance matrix with respect to elevation.

Without loss of generality, the estimated state covariance matrix of KF at time instance $k$ can be written as [16]

$$
\mathbf{P}_{k}=\left(\mathbf{I}-\mathbf{K}_{k} \mathbf{H}_{k}\right) \mathbf{P}_{k, k-1}
$$

where $\mathbf{K}_{k}$ denotes the gain matrix at $k, \mathbf{H}_{k}$ denotes the measurement matrix, and $\mathbf{P}_{k, k-1}$ denotes the predicted state covariance from $k-1$ to $k$. The derivative of (56) with respect to elevation $\varepsilon_{k}$ can be written as

$$
\begin{aligned}
\frac{\partial \mathbf{P}_{k}}{\partial \varepsilon_{k}} & =-\mathbf{H}_{e q} \mathbf{P}_{k, k-1}+\left(\mathbf{I}-\mathbf{K}_{k} \mathbf{H}_{k}\right) \frac{\partial \mathbf{P}_{k, k-1}}{\partial \varepsilon_{k}} \\
\mathbf{H}_{e q} & =\left[\frac{\partial \mathbf{K}_{k}}{\partial \varepsilon_{k}} \mathbf{H}_{k}+\mathbf{K}_{k} \frac{\partial \mathbf{H}_{k}}{\partial \varepsilon_{k}}\right]
\end{aligned}
$$

The detailed derivations of (57) are shown in Appendix C.

According to (44), the partial derivatives of the state covariance are continuous. Theoretically, the estimated state covariance obtained by KF has the minimum values when the elevation used in the model is the true value $\varepsilon_{A_{-} E N U}$. Or else, the additional error covariance can be written as

$$
\Delta \mathbf{P}_{k} \approx\left|\frac{\partial \boldsymbol{P}_{k}}{\partial \varepsilon_{k}} \Delta \varepsilon_{k}\right|>\mathbf{0}
$$

then the additional errors of the estimation $\widehat{\boldsymbol{\beta}}(k)$ caused by the elevation errors can be denoted approximately by

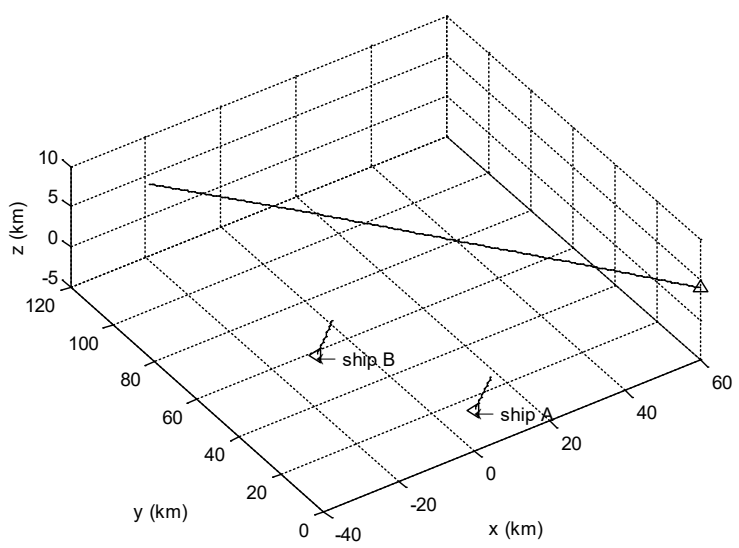

FIGURE 5: The geometry of radar and target.



(a) Range bias

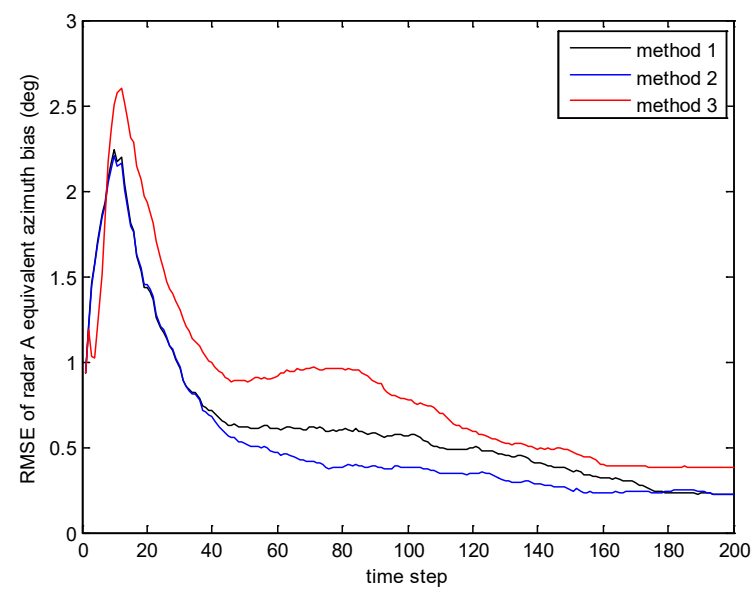

(b) Azimuth bias 




(c) Elevation bias

FIGURE 6: RMSEs of radar A gross bias estimations.

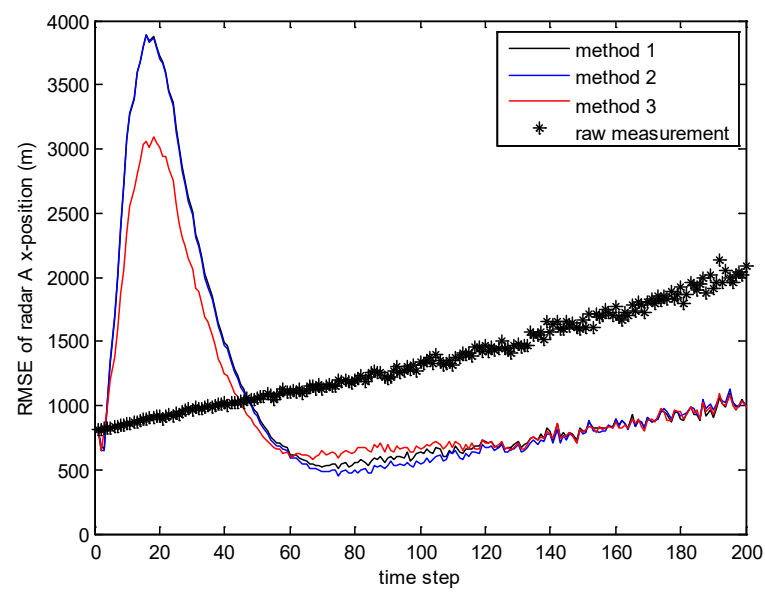

(a) $x$-coordinates

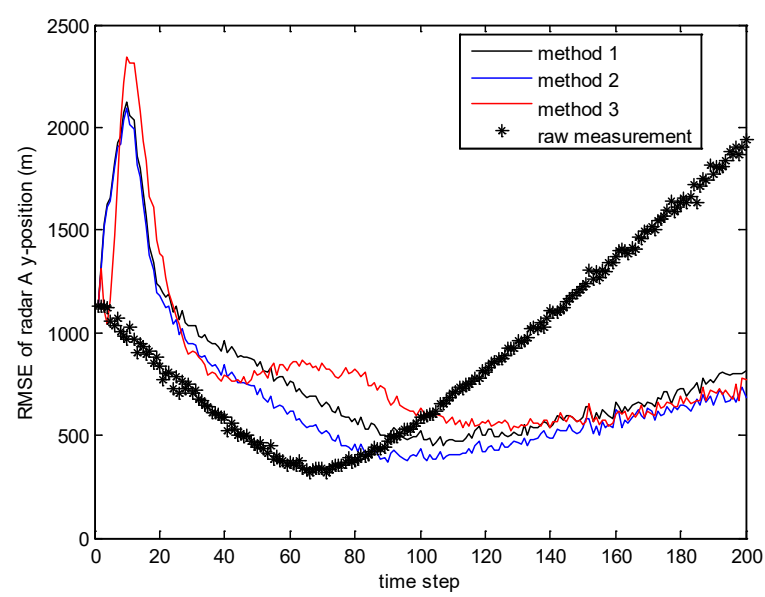

(b) $y$-coordinates

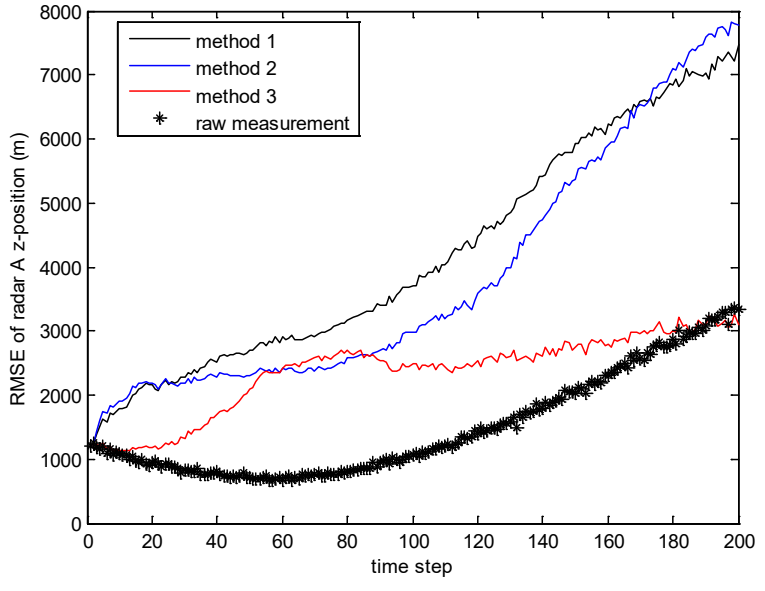

(c) $z$-coordinates

FIGURE 7: RMSEs of the target location in $x y z$-coordinates after rectifying radar A measurements by bias estimations.

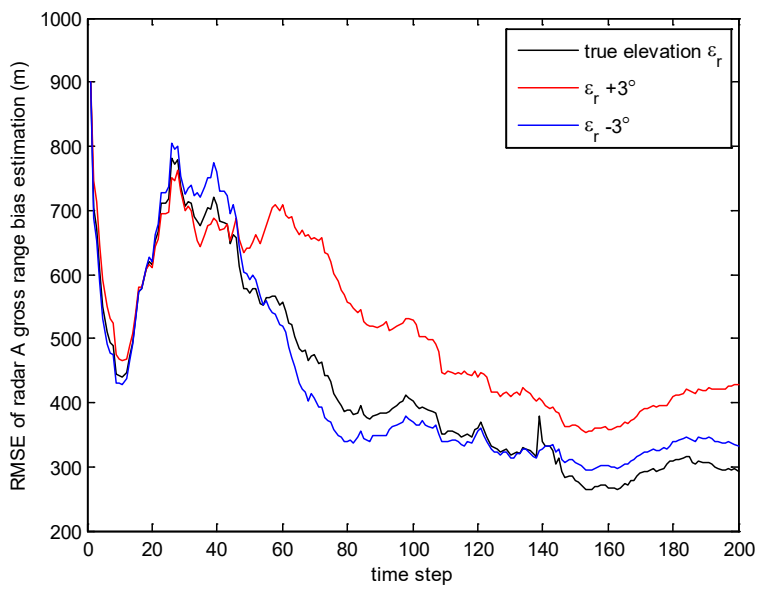

(a) Radar A gross range estimation

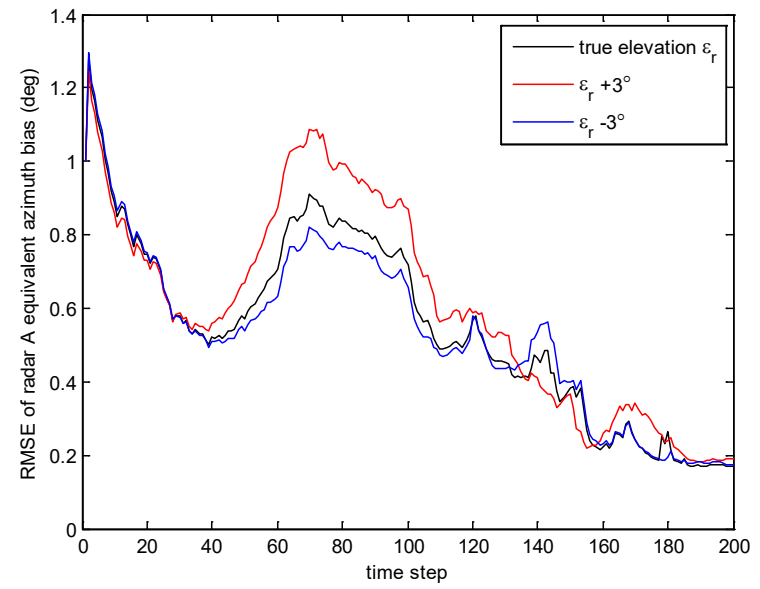

(b) Radar A equivalent azimuth estimation

FIGURE 8: RMSEs of radar bias estimation. 


$$
\widetilde{\boldsymbol{\beta}}(k)=\sqrt{\operatorname{diag}\left(\mathbf{P}_{k}+\left|\frac{\partial \boldsymbol{P}_{k}}{\partial \varepsilon_{k}} \Delta \varepsilon_{k}\right|\right)}-\sqrt{\operatorname{diag}\left(\mathbf{P}_{k}\right)}
$$

where $\operatorname{diag}(\mathbf{M})$ denotes the diagonal elements of the matrix $\mathbf{M}$, and $\sqrt{\mathbf{V}}$ denotes the square root of each element of vector V.

\section{Simulation and results}

In this section, two experiments will be presented. For the first experiment, a 3-D and 2-D radar network is simulated and three methods proposed in Section 3 are compared respectively. For the second experiment, method 3 is applied for a 2-D radar network, and the bias estimation errors caused by the target altitude errors are given by using sensitivity analysis theory. The only difference between both experiments is that the latter uses a 2-D radar to replace the 3D radar of the first experiment. The 2-D radar B in both experiments has the same parameters.

In the simulated scenario, a common track is generated for two radars installed on different ships. Assuming that ship A and $\mathrm{B}$ are moving with constant velocity model, and the initial geographical coordinates are $\left[40^{\circ}, 116^{\circ}, 10 \mathrm{~m}\right],\left[40.4^{\circ}, 115.8^{\circ}\right.$, $10 \mathrm{~m}]$, respectively. The initial states of both ships in their native ENU frame are $[0,10 \mathrm{~m} / \mathrm{s}, 0,10 \mathrm{~m} / \mathrm{s}, 0,0]$.

In the state vector, the variables denote the position and velocity components of the target in $x$ (east), $y$ (north), and $z$ (up) coordinates, respectively.

The standard deviations of both ships' process noise are equal which are given in $x, y$, and $z$ coordinates by $0.1 \mathrm{~m} / \mathrm{s}^{2}$, $0.1 \mathrm{~m} / \mathrm{s}^{2}$, and $0 \mathrm{~m} / \mathrm{s}^{2}$, respectively. Fusion center locates at the initial position of ship A. Constant velocity model is also used for the target. The initial state of the target in fusion center is [60 km, $-80 \mathrm{~m} / \mathrm{s}, 0 \mathrm{~km}, 120 \mathrm{~m} / \mathrm{s}, 4 \mathrm{~km}, 1 \mathrm{~m} / \mathrm{s}]$. The standard deviations of the process noises in $x, y$, and $z$ coordinates are set to $1 \mathrm{~m} / \mathrm{s}^{2}, 1 \mathrm{~m} / \mathrm{s}^{2}$, and $0.1 \mathrm{~m} / \mathrm{s}^{2}$, respectively.

The geometry of radar and the target is shown in Fig.5. The true offset biases of both radars are assumed to be constant and equal as $\Delta r_{i}=300 \mathrm{~m}, k_{r i}=0.01, \Delta \theta_{i}=2^{\circ}$, and $\Delta \varepsilon_{A}=2^{\circ}$, respectively. The standard deviations of random measurement noises are $\sigma_{r A}=50 \mathrm{~m}, \sigma_{\theta_{A}}=0.5^{\circ}, \sigma_{\varepsilon_{A}}=$ $0.5^{\circ}, \sigma_{r B}=200 \mathrm{~m}$, and $\sigma_{\theta_{B}}=0.5^{\circ}$, respectively. The attitude biases of both platforms are also assumed to be constant and equal as $\Delta \phi_{i}=\Delta \eta_{i}=\Delta \psi_{i}=1^{\circ}$. It is assumed that both radars are synchronized with the same sampling intervals $T R_{s p}=5 \mathrm{~s} .200$ scans of the target are simulated and the number of Monte Carlo runs is set to 100 .

Figures 6 and 7 contain all the simulation results.

In order to calculate the covariance matrix of the state vector $\boldsymbol{\beta}$, it is assumed that the maximum magnitudes are 2 degrees for each attitude bias of both platforms, and the standard deviation for velocities in $x, y$, and $z$ directions are $200 \mathrm{~m} / \mathrm{s}, 200 \mathrm{~m} / \mathrm{s}$, and $20 \mathrm{~m} / \mathrm{s}$, respectively.

For the second experiment, in each KF computation cycle, three different elevation values are used for comparison, that is, the true elevation value $\varepsilon_{A_{-} E N U}(k), \varepsilon_{A_{-} E N U}(k)+3^{\circ}$, and $\varepsilon_{A_{-} E N U}(k)-3^{\circ}$. Figures 8-10 contain all the simulation results.

For length limitation, the results of radar B will not be given.

Figure 6 present the root mean square errors (RMSE) of radar A gross bias estimations. (a) represent the range biases. Here, "gross" means the sum of the range bias and the range bias caused by the gain of the range; (b) present the azimuth biases; and (c) present the elevation biases. For (b) and (c), "gross" means the sum of the offset bias and the bias caused by the attitude biases. In Figs. 6-7, the black, blue, and red lines represent the results of method 1, method 2, and method 3 , respectively.

Figure 7 are the RMSEs of the rectified measurements in each axis of ENU frame, where the raw measurements are rectified by the gross bias estimation directly. (a), (b), and (c) present $x$-, $y$-, and $z$ - coordinates, respectively. The asteroids represent radar raw measurements.

For the second experiment, Fig. 8 presents the RMSEs of radar A bias estimation. The black, blue, and red lines represent the estimation results using the true elevation values $\varepsilon_{A_{-} E N U}(k), \varepsilon_{A_{-} E N U}(k)+3^{\circ}$, and $\varepsilon_{A_{-} E N U}(k)-3^{\circ}$, respectively.

Figure 9 represent the RMSEs of radar raw measurements rectified by different systematic bias estimations, the asterisk signs in the figures represent the curves of raw measurements. 


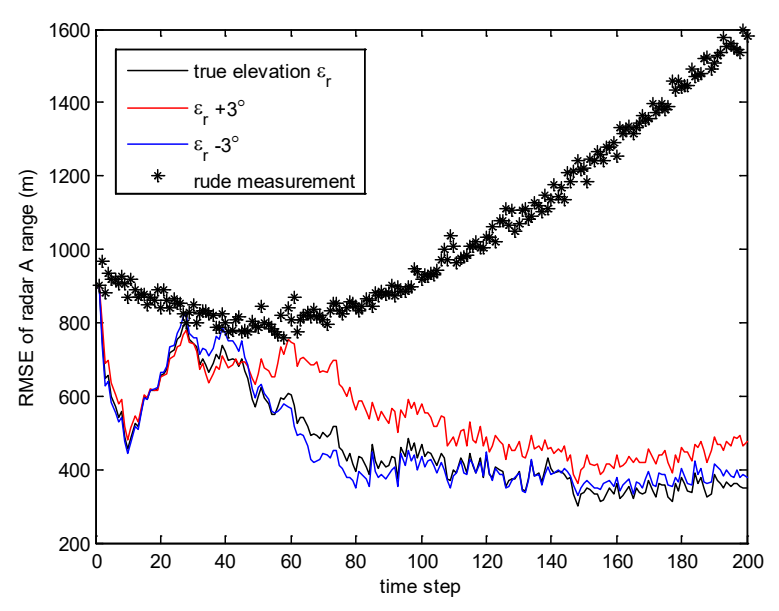

(a) Radar A gross range estimation

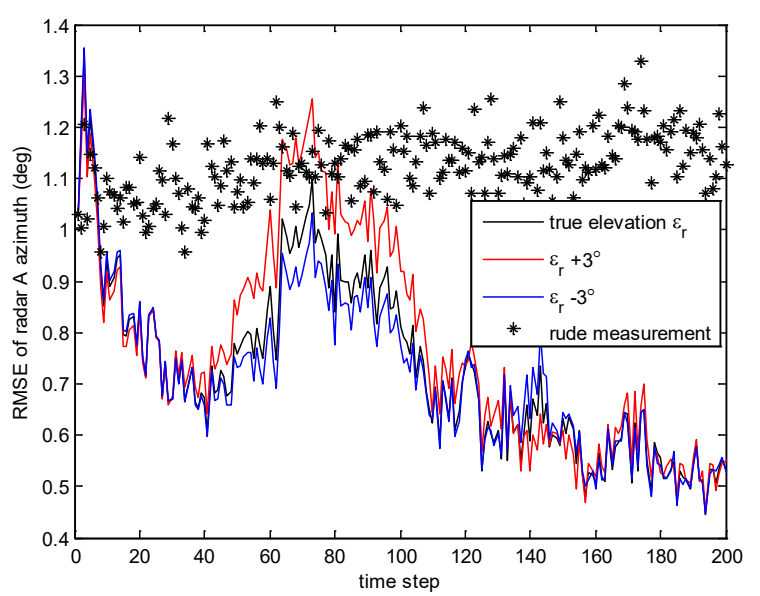

(b) Radar A equivalent azimuth estimation

FIGURE 9: RMSEs of 2-D radar A measurement rectified by bias estimations.



(a) Radar A range bias

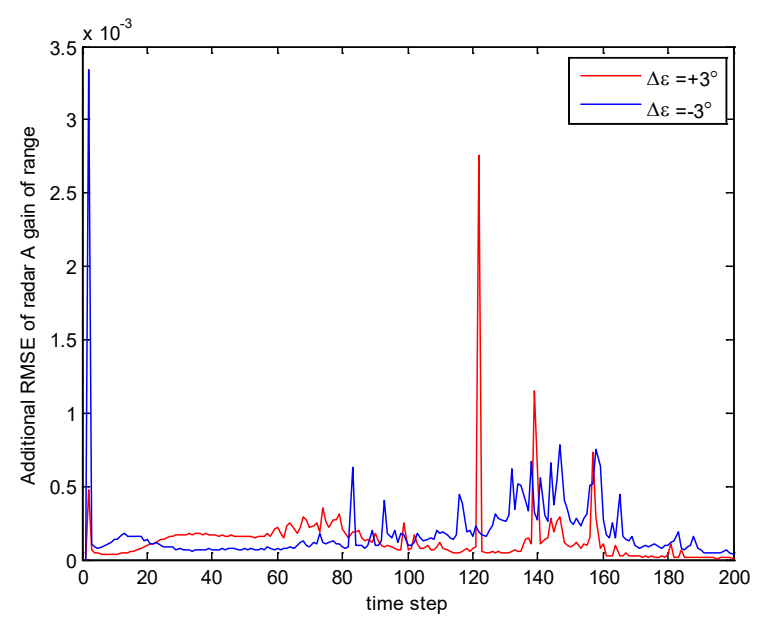

(b) Radar A gain of range

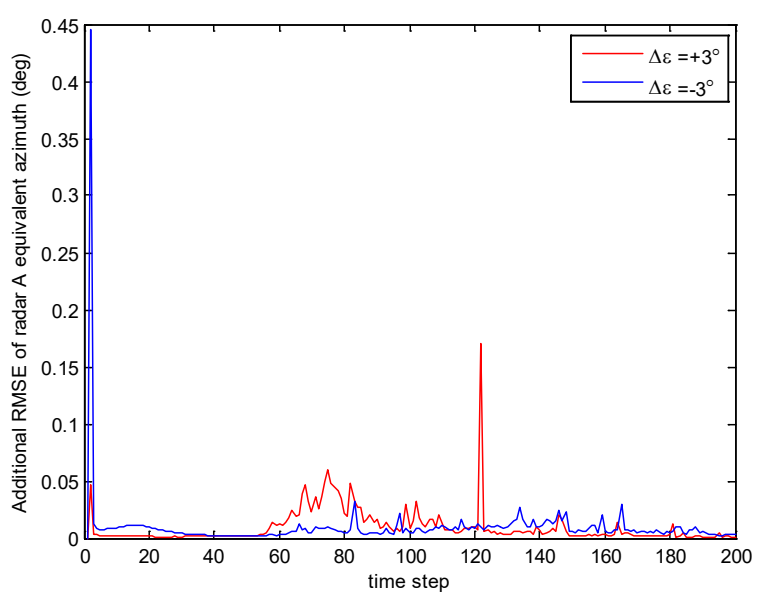

(c) Radar A equivalent azimuth bias

FIGURE 10: The additional errors of the bias estimations caused by the elevation errors.

Figure 10 present the theoretical computations of additional bias estimation errors caused by the elevation errors by using (61). Since the estimated error covariance matrix converges gradually, the effects will decrease gradually, too.

Though the simulation results of the sensitivity analysis in Fig.10 show that the elevation errors have minor influences on the bias estimations, in fact, Fig. 6 and Fig. 8 show that their effects cannot be omitted because for radar A, The reference point corresponding to the preset elevation value instead of the true target location is used to establish the registration equations, the errors between them will be embodied completely in the estimations of range and azimuth biases. The bigger the errors, the worse the estimate performances.

Comparing these figures, the following results can be 
obtained: (1) all the three methods in the paper can be applied for the systematic biases estimation; (2) since the target altitude information can only be obtained by 3 -D radar, and it contains errors, the elevation bias estimation results and the rectified radar measurement errors in $\mathrm{z}$ - direction have big errors;

(3) The registration equations are established based on the fact that the true target coordinates obtained by two different radar measurements are equal, however, since 2-D radar does not have complete target information, it is the reference points instead of the true target are used to establish the registration equations. In fact, three methods in the paper correspond to two different ways of selecting reference points. The errors between the reference point and the true target will be embodied in all the bias estimation errors. In view of this, none of the three methods outperforms the others in all the situations, and each method will perform best in certain circumstances. If the apriori ${ }^{\text {iv }}$ altitude information is more accurate and can be obtained, it will be used as the most preferable method.

\section{Summary and Conclusions}

The registration methods for mobile 3-D and 2-D radar network were proposed in the paper. These methods can also be applied for 2-D radar network. Since the attitude biases are dependent with radar offset biases, according to the observability criterion, attitude biases are merged into radar offset bias to be estimated.

Since the target altitudes cannot be obtained by 2-D radar which are needed to establish the registration equations, three methods are given in the paper according to different methods of setting the altitudes. These methods always introduce unavoidable errors in the registration equations, and the errors will be embodied in all the bias estimation results. In view of this, all the three methods can be used and only one method will perform best in certain situations. If more accurate apriori target altitude information can be obtained, it can be employed and yield better registration results.

It should be noted that the variations of the equivalent biases (converted from the attitude biases) caused by the variations of the target locations are small and can be viewed as process noises are conditioned on single target situation, as for dispersed targets, these assumptions will not hold and the methods in the paper should be revised, which will be discussed in the future.

\section{Acknowledgements}

This work was supported in part by the National Natural Science Foundation of China 61032001, 61102165, 61002006 and Special Foundation Program for Mountain Tai Scholars of China.

\section{Appendix A: Proof of unobservability of registration equations when offset and attitude biases occur as state variables simultaneously}

Attitude biases can be converted to measurement errors in the form of (1)-(3). And the equivalent measurement errors (converted from the attitude biases) and offset biases always occur simultaneously in the addition form in the registration equations. Since the target coordinates variations are small compared with their coordinates at consecutive measurement instance, according to (1)-(3), the coefficients of attitude bias variables can be approximated as constants, and then the coefficients of attitude and offset biases in each registration equation have the same ratio.

In [19] Lemma 1, they testified that the hybrid initial state of a discrete-time linear hybrid system is observable if and only if it is distinguishable. And the 'indistinguishability' was defined by Definition 3 as: A discrete-time linear hybrid system is indistinguishable on $\left[k_{0}, k_{0}+k\right]$ if there exist output sequence $Y_{k}$ and $Y_{k}^{\prime}$ on $k \in\left[k_{0}, k_{0}+k\right]$ starting from any two different hybrid states $\left(q_{k_{0}}, x_{k_{0}}\right)$ and $\left(q_{k_{0}}^{\prime}, x_{k_{0}}^{\prime}\right)$, whose covariances are equal.

So, in this part, the results above will be used to prove that the registration equations are unobservable when two state variables' coefficients have the same ratio in each registration equation.

Without loss of generality, assuming that the registration equations contain three uncorrelated variables, $x_{1}, x_{2}$, and $x_{3}$, respectively. The coefficient ratio of $x_{2}$ to $x_{1}$ is $\alpha$. The registration equations can be written as:

$$
\mathbf{Y}=\mathbf{F}\left(x_{1}, x_{2}, x_{3}\right)=\mathbf{F}\left(x_{1}+\alpha x_{2}, x_{3}\right)
$$


Assuming $\left(\hat{x}_{1}, \hat{x}_{2}, \hat{x}_{3}\right)$ is a solution of $(\mathrm{A} 1)$, that is,

$$
\begin{array}{r}
\mathbf{Y}_{1}=\mathbf{F}\left(\hat{x}_{1}, \hat{x}_{2}, \hat{x}_{3}\right)=\mathbf{F}\left(\hat{x}_{1}+\alpha \hat{x}_{2}, \hat{x}_{3}\right) \\
\mathbf{Y}_{2}=\mathbf{F}\left(\hat{x}_{1}+d, \hat{x}_{2}-d / \alpha, \hat{x}_{3}\right)=\mathbf{F}\left[\hat{x}_{1}+d+\alpha\left(\hat{x}_{2}-d / \alpha\right), \hat{x}_{3}\right]=\mathbf{F}\left(\hat{x}_{1}+\alpha \hat{x}_{2}, \hat{x}_{3}\right)=\boldsymbol{Y}_{1}(\mathrm{~A}
\end{array}
$$
since where $d(\neq 0)$ is an arbitrary real.

Since $x_{1}$ and $x_{2}$ always occur simultaneously in the form of addition in the registration equations, that is

$$
\frac{\partial \mathbf{F}}{\partial x_{1}}=\frac{\partial \mathbf{F}}{\partial\left(\alpha x_{2}\right)}
$$

Then the variance of $\mathbf{Y}_{1}$ and $\boldsymbol{Y}_{2}$ (first-order approximation) can be written respectively as:

$$
D\left(\mathbf{Y}_{1}\right)=\frac{\partial \mathbf{F}}{\partial \hat{x}_{1}} D\left(\hat{x}_{1}+\alpha \hat{x}_{2}\right)\left(\frac{\partial \mathbf{F}}{\partial \hat{x}_{1}}\right)^{\dagger}+\frac{\partial \mathbf{F}}{\partial \hat{x}_{3}} D\left(\hat{x}_{3}\right)\left(\frac{\partial \mathbf{F}}{\partial \hat{x}_{3}}\right)^{\dagger}
$$

$$
D\left(\mathbf{Y}_{2}\right)=\frac{\partial \mathbf{F}}{\partial \hat{x}_{1}} D\left[\hat{x}_{1}+d+\alpha\left(\hat{x}_{2}-d / \alpha\right)\right]\left(\frac{\partial \mathbf{F}}{\partial \hat{x}_{1}}\right)^{\dagger}+\frac{\partial \mathbf{F}}{\partial \hat{x}_{3}} D\left(\hat{x}_{3}\right)\left(\frac{\partial \mathbf{F}}{\partial \hat{x}_{3}}\right)^{\dagger}=D\left(\mathbf{Y}_{1}\right)
$$

where $D(\mathbf{Y})$ represents the variance of $\boldsymbol{Y}$.

According to the 'indistinguishability' definition in [19] and (A6), the registration equations (A1) is indistinguishable. According to Lemma 1 in [19], (A1) is unobservable.

Reviewing the proof process above, if (A1) is a deterministic system, (A3) demonstrates that the states can Not be uniquely determined from one output and (A1) is unobservable. If (A1) is a stochastic system and only the output $\boldsymbol{Y}$ contains random noises, just like the first-order linearized registration equations in the article, (A1) is unobservable.

So, from the perspective of OM as well as the concept of "indistinguishability", we both proved that the registration equations are unobservable when two variables' coefficients have the same ratio in every equation and both variables occur simultaneously in the form of addition in the registration equations.

Furthermore, according to the relationship between $x_{1}$ and $x_{2}$, it is convenient to merge them into a new variable. Then the registration equations only contain 'offset biases' in form, and the system is observable. The estimate of 'offset biases' can be used to eliminate both attitude and offset biases simultaneously. It seems that there is unnecessary to estimate both kinds of biases separately.

\section{Appendix B: The derivation of target altitude in 2-D radar ENU frame}

Omitting the influences of attitude biases and random noises, the approximate target coordinates in radar A ENU frame can be written as

$$
\mathbf{X}_{A_{-} E N U}^{r}(k)=\left[\begin{array}{l}
x_{A_{-} E N U}^{r}(k) \\
y_{A_{-} E N U}^{r}(k) \\
z_{A_{-} E N U}^{r}(k)
\end{array}\right]=r_{A}(k) \mathbf{e}\left(\alpha_{A}, \varepsilon_{A}\right)(k)
$$

where the superscript ' $r$ ' denotes the variable is obtained by using radar measurements only.

According to (7), the ECEF coordinates can be written as

$$
\mathbf{X}_{A_{-} E C E F}^{r}(k)=\mathbf{X}_{A S}(k)+\mathbf{T}_{A}(k) \mathbf{X}_{A_{-} E N U}^{r}(k)
$$

According to (8a), the ENU coordinates of radar B can be written as

$$
\mathbf{X}_{B_{-} E N U}^{A r}(k)=\left[x_{B_{-} E N U}^{A r}(k), y_{B_{-} E N U}^{A r}(k), z_{B_{-} E N U}^{A r}(k)\right]^{\dagger}
$$

or

$$
\mathbf{X}_{B_{-} E N U}^{A r}(k)=\mathbf{T}_{B}^{-1}(k)\left[\mathbf{X}_{A_{-} E C E F}^{r}(k)-\mathbf{X}_{B S}(k)\right]
$$

In fact, this method uses the biased radar raw measurements to obtain the approximate target altitudes in radar B local ENU frame.

\section{Appendix C: Sensitivity analysis of elevation}

Without loss of generality, assuming the dynamic equations of discrete system can be written as

$$
\begin{aligned}
& \mathbf{X}_{k}=\boldsymbol{\phi}_{k, k-1} \mathbf{X}_{k-1}+\Gamma_{k-1} \mathbf{W}_{k-1} \\
& \mathbf{Z}_{k}=\mathbf{H}_{k} \mathbf{X}_{k}+\mathbf{G}_{k} \mathbf{V}_{k}
\end{aligned}
$$

where $\boldsymbol{\phi}_{k, k-1}$ denotes state transition matrix from time $k$-1 to $k, \mathbf{H}_{k}$ denotes the measurement matrix, $\mathbf{W}_{k}$ and $\mathbf{V}_{k}$ denote the process noise and measurement noise, respectively. They are Gaussian white noises with covariance matrix 
denoted as $\mathbf{Q}_{k}$ and $\mathbf{R}_{k}$, respectively.

$$
\frac{\partial \mathbf{P}_{k}}{\partial b_{k}}=-\mathbf{H}_{e q} \mathbf{P}_{k, k-1}+\left(\mathbf{I}-\mathbf{K}_{k} \mathbf{H}_{k}\right) \frac{\partial \mathbf{P}_{k, k-1}}{\partial b_{k}}
$$

$\mathrm{KF}$ equations can be written as

$$
\begin{aligned}
& \mathbf{P}_{k, k-1}=\boldsymbol{\phi}_{k, k-1} \mathbf{P}_{k-1} \boldsymbol{\phi}_{k, k-1}^{\dagger}+\boldsymbol{\Gamma}_{k-1} \mathbf{Q}_{k-1} \boldsymbol{\Gamma}_{k-1}^{\dagger} \\
& \mathbf{K}_{k}=\mathbf{P}_{k, k-1} \mathbf{H}_{k}^{\dagger}\left(\mathbf{H}_{k} \mathbf{P}_{k, k-1} \mathbf{H}_{k}^{\dagger}+\mathbf{G}_{k} \mathbf{R}_{k} \mathbf{G}_{k}^{\boldsymbol{T}} \equiv \mathbf{M}_{k}\right)^{-\mathbf{1}}(\mathrm{C} 4) \\
& \mathbf{P}_{k}=\left(\mathbf{I}-\mathbf{K}_{k} \mathbf{H}_{k}\right) \mathbf{P}_{k, k-1}
\end{aligned}
$$$$
\mathbf{H}_{e q}=\frac{\partial \mathbf{K}_{k}}{\partial b_{k}} \mathbf{H}_{k}+\mathbf{K}_{k} \frac{\partial \mathbf{H}_{k}}{\partial b_{k}}
$$

Since

$$
\frac{\partial \mathbf{P}_{k, k-1}}{\partial b_{k}}=0, \frac{\partial \mathbf{R}_{k}}{\partial b_{k}}=0
$$

Assuming a perturbation variable $b_{k}$ is included in (C5), then the derivative of $(\mathrm{C} 5)$ with respect to $b_{k}$ can be written the derivative of $(\mathrm{C} 4)$ can be written as as

$$
\begin{aligned}
& \frac{\partial \mathbf{K}_{k}}{\partial b_{k}}=\frac{\partial \mathbf{P}_{k, k-1}}{\partial b_{k}} \mathbf{H}_{k}^{\dagger} \mathbf{M}_{k}^{-1}+\mathbf{P}_{k, k-1} \frac{\partial \mathbf{H}_{k}^{\dagger}}{\partial b_{k}} \mathbf{M}_{k}^{-1}+\mathbf{P}_{k, k-1} \mathbf{H}_{k}^{\dagger} \frac{\partial \mathbf{M}_{k}^{-\mathbf{1}}}{\partial b_{k}} \\
& \frac{\partial \mathbf{M}_{k}^{-1}}{\partial b_{k}}=\left(\frac{\partial \mathbf{H}_{k}}{\partial b_{k}} \mathbf{P}_{k, k-1} \mathbf{H}_{k}^{\dagger}+\mathbf{H}_{k} \frac{\partial \mathbf{P}_{k, k-1}}{\partial b_{k}} \mathbf{H}_{k}^{\dagger}+\mathbf{H}_{k} \mathbf{P}_{k, k-1} \frac{\partial \mathbf{H}_{k}^{\dagger}}{\partial b_{k}}+\frac{\partial \mathbf{G}_{k}}{\partial b_{k}} \mathbf{R}_{k} \mathbf{G}_{k}^{\dagger}+\mathbf{G}_{k} \mathbf{R}_{k} \frac{\partial \mathbf{G}_{k}^{\dagger}}{\partial b_{k}}\right)^{-1} \\
& \frac{\partial \mathbf{P}_{k, k-1}}{\partial b_{k}}=\frac{\partial \boldsymbol{\phi}_{k, k-1}}{\partial b_{k}} \mathbf{P}_{k-1} \boldsymbol{\phi}_{k, k-1}^{\dagger}+\boldsymbol{\phi}_{k, k-1} \mathbf{P}_{k-1} \frac{\partial \boldsymbol{\phi}_{k, k-1}^{\dagger}}{\partial b_{k}}+\frac{\partial \mathbf{\Gamma}_{k-1}}{\partial b_{k}} \mathbf{Q}_{k-1} \boldsymbol{\Gamma}_{k-1}^{\dagger}+\boldsymbol{\Gamma}_{k-1} \mathbf{Q}_{k-1} \frac{\partial \mathbf{\Gamma}_{k-1}^{\dagger}}{\partial b_{k}}
\end{aligned}
$$

For the registration models composed of (29), (31), and (35), we have

$$
\boldsymbol{\phi}_{k, k-1}=\mathbf{I}, \boldsymbol{\Gamma}_{k-1}=\mathbf{0}
$$

where $\mathbf{0}$ denotes the corresponding zero matrix; i.e., e matrix whose elements are all zero. Then,

$$
\begin{gathered}
\frac{\partial \boldsymbol{\Phi}_{k, k-1}}{\partial \varepsilon_{A_{-} E N U}(k)}=\mathbf{0}, \frac{\partial \Gamma_{k-1}}{\partial \varepsilon_{A_{-} E N U}(k)}=\mathbf{0} \\
\frac{\partial \mid \begin{array}{l}
\mathbf{H}_{k} \\
\mathbf{G}_{k}
\end{array}}{\partial \varepsilon_{k}}(1,:)=\left\{\left[\mathbf{X}_{B S}(k)-\mathbf{X}_{A s}(k)\right]^{\dagger} \mathbf{T}_{A}(k) \frac{\partial \mid \begin{array}{l}
\mathbf{A}_{A}(k) \\
\mathbf{C}_{A}(k)
\end{array}}{\partial \varepsilon_{k}}, 0,0,0\right\}
\end{gathered}
$$

$$
\begin{gathered}
\frac{\left.\partial\right|_{\mathbf{G}_{k}} ^{\mathbf{H}_{k}}}{\partial \varepsilon_{k}}(2,:)=r^{-1} \mid \begin{array}{l}
\mathbf{X}_{1}-\left.r^{-3}\right|_{\mathbf{y}_{1}} ^{\mathbf{X}_{3}}-\left.r^{-5}\right|_{\mathbf{y}_{3}} ^{\mathbf{X}_{5}} \\
\mathbf{y}_{5}
\end{array} r^{2}=\left(x_{B_{-} E N U}^{A} \equiv x\right)^{2}(k)+\left(y_{B_{-} E N U}^{A} \equiv y\right)^{2}(k) \\
\dot{x}_{\mathbf{w}}^{\boldsymbol{\beta}}=\frac{\partial x_{B_{-} E N U}^{A}(k)}{\left.\partial\right|_{\mathbf{w}_{A}} ^{\boldsymbol{\beta}_{A}}}, \dot{x}_{\varepsilon}=\frac{\partial x_{B_{-} E N U}^{A}(k)}{\partial \varepsilon_{k}}, \dot{y}_{\left.\right|_{\mathbf{w}} ^{\beta}}=\frac{\partial y_{B_{-} E N U}^{A}(k)}{\left.\partial\right|_{\mathbf{w}_{A}} ^{\boldsymbol{\beta}_{A}}}, \dot{y}_{\varepsilon}=\frac{\partial y_{B_{-} E N U}^{A}(k)}{\partial \varepsilon_{k}}
\end{gathered}
$$

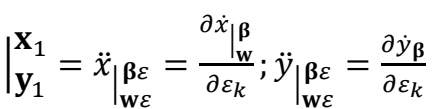

$$
\left.\right|_{\mathbf{y}_{3}} ^{\mathbf{x}_{3}}=y \dot{y}_{\varepsilon} \dot{x}_{\left.\right|_{\mathbf{w}} ^{\boldsymbol{\beta}}}+x \dot{x}_{\varepsilon} \dot{x}_{\left.\right|_{\mathbf{w}} ^{\boldsymbol{\beta}}}+2 x \dot{x}_{\varepsilon} \dot{x}_{\left.\right|_{\mathbf{w}} ^{\boldsymbol{\beta}}}+\left.x^{2}\right|_{\mathbf{y}_{1}} ^{\mathbf{x}_{1}}+y \dot{x}_{\varepsilon} \dot{y}_{\left.\right|_{\mathbf{w}} ^{\boldsymbol{\beta}}}+x \dot{y}_{\varepsilon} \dot{y}_{\left.\right|_{\mathbf{w}} ^{\boldsymbol{\beta}}}+x y \ddot{y}_{\left.\right|_{\mathbf{w} \varepsilon} ^{\boldsymbol{\beta} \varepsilon}}
$$

$$
\left.\right|_{\mathbf{y}_{5}} ^{\mathbf{x}_{5}}=3\left(y \dot{y}_{\varepsilon}+x \dot{x}_{\varepsilon}\right)\left(x^{2} \dot{x}_{\left.\right|_{\mathbf{w}} ^{\boldsymbol{\beta}}}+x y \dot{y}_{\left.\right|_{\mathbf{w}} ^{\boldsymbol{\beta}}}\right)
$$

given.

Substituting (C7)-(C14) into (C6), the partial derivative of the state covariance matrix with respect to elevation can be 


\section{References}

[1] G.H. Wang, L. Chen, S.Y. Jia, "Optimized bias estimation model for 3-D radar considering platform attitude errors," IEEE Sys. Mag., vol. 27, no. 1, pp. 19-26, 2012, DOI: http://doi.org/10.1109/MAES.2012.6145437.

[2] N. Okello, B. Ristic, "Maximum likelihood registration for multiple dissimilar sensors," IEEE Tran. AES, vol. 39, no. 3, pp.1074-1083, July2003, DOI: http://doi.org/10.1109/TAES.2003.1238759.

[3] D.F. Crouse, R.W. Osborne, III, K. Pattipati, P. Willett, Y. Bar-Shalom, "Efficient 2D sensor location estimation using targets of opportunity," Journal of Advances in Information Fusion, vol. 8, no. 1, June, 2013, pp. 73-89.

[4] G.H. Wang, L. Chen, S.Y. Jia, I. Progri, “Optimized Bias Estimation Model for Mobile Radar Error Registration," The J. of Navigation, vol. 66, 227-248, 2013, DOI: http://doi.org.ezproxy.lib.usf.edu/10.1017/S0373463312 000458.

[5] L. Chen, G.H. Wang, I.F. Progri, "Quaternionic analysis of the influences of systematic biases on radar data processing," J. Geol. Geoinfo. Geointel., vol. 2015, Article ID 2015072501, 18 pg., Nov. 2015, DOI: http://doi.org/10.18610/JG3.2015.072501, http://giftet.com/JG3/2015/072501.pdf.

[6] L. Chen, G.H. Wang, I.F. Progri, "Unified Registration Model for both stationary and mobile 3-D radar alignment," Journal of Electrical \& Computer Engineering, vol. 2014, Article ID 598463, DOI: http://doi.org/10.1155/2014/598463.

[7] L. Chen, G. H. Wang, S. Y. Jia, I. Progri, “Attitude bias conversion model for mobile radar error registration," The J. of Navigation, vol. 65, no. 4, pp. 651-670, DOI: http://doi.org.ezproxy.lib.usf.edu/10.1017/S0373463312 000239.

[8] A.D. King, "Inertial navigation - past, present, and future," IEE Colloquium, Savoy Place, London WC2R OBL, UK, pp.3/1-3/9, 1997.

[9] U.I. Bhatti, W.Y. Ochieng, "Failure modes and models for integrated GPS/INS systems," The J. of Navigation, vol. 60, no. 2, pp. 327-348, May 2007, DOI:
http://doi.org.ezproxy.lib.usf.edu/10.1017/S0373463307 004237.

[10] Y.F. Zhou,L. Henry, B. Martin, "Sensor alignment with Earth-centered Earth-Fixed coordinate system," IEEE Tran. AES, vol. 35, no. 2, pp. 410-416, Feb. 1999.

[11] M. Petrou, "Image registration: an overview," Advances in imaging and electron physics, vol. 130. pp. 243-291, 2004, DOI: http://doi.org/10.1016/S10765670(04)30003-0.

[12]F. Simonot, F. Simonot-Lion, and Y.Q. Song, "Dependability evaluation of real-time applications distributed on TDMA-based networks," in Proc. $5^{\text {th }}$ IF AC Intr. Conf. on Fieldbus Sys. \& their App., Puebla, Mexico, Nov., 2005.

[13] J.M. Holtzman, “On using perturbation analysis to do sensitivity analysis: derivatives versus differences," IEEE Trans. Aut. Con., vol. 37, no. 2, pp. 243-247, 1992, DOI: http://doi.org/10.1109/9.121628.

[14] Y.Y. Qin, H.Y. Zhang, S.H. Wang, Kalman Filter and Integrated Navigation Theory. [M]. Xi'an: Northwestern Polytechnic University Press, 1998.

[15] G.H. Wang, Study on Key Techniques in Radar Netting, Postdoctoral Reports, Nanjing Research Institute of Electronics Technology, Nanjing, May, 2004.

[16] Y. He, J.J. Xiu, J.W. Zhang, and X. Guan, Radar Data Processing With Applications (second edition) Publishing House of Electronics Industry, Beijing, China, pp.31-35, 2009, (in Chinese).

[17] Anon., "Fleming's left-hand rule for motors," From Wikipedia, the free encyclopedia, Jun., 2018. https://en.wikipedia.org/wiki/Fleming\%27s_lefthand_rule_for_motors.

[18] Anon., "Right-hand rule," From Wikipedia, the free encyclopedia, Jun., 2018, https://en.wikipedia.org/wiki/Right-hand_rule.

[19] I. Hwang, H. Balakrishnan, C. Tomlin "Observability criteria and estimator design for stochastic linear hybrid systems," in Proc. European Control Conference (ECC), 2003, Cambridge, UK, pp. 3317-3322, Sep. 2003, URL. http://web.mit.edu/hamsa/www/pubs/ECC03_HBT.pdf

\footnotetext{
iii Units of $h_{s}$ are in $\mathrm{m}$.

iv Apriori means relating to or denoting reasoning or knowledge which
}

\footnotetext{
'The superscript ' $\dagger$ ' denotes matrix or vector transposition.

iiUnits of $r$ are in $\mathrm{km}$.
} 
postulated, or self-evident.

proceeds from theoretical deduction rather than from observation or experience, such as theoretical, deduced, deductive, inferred, scientific, 